\title{
Session-based Social and Dependency-aware Software Recommendation
}

\author{
Dengcheng Yan ${ }^{\mathrm{a}}$, Tianyi Tang ${ }^{\mathrm{a}}$, Wenxin Xie ${ }^{\mathrm{a}}$, Yiwen Zhang ${ }^{\mathrm{a}, *}$, Qiang $\mathrm{He}^{\mathrm{b}}$ \\ ${ }^{a}$ School of Computer Science and Technology, Anhui University, Hefei 230601, China \\ ${ }^{b}$ School of Software and Electrical Engineering, Swinburne University of Technology, Melbourne \\ 3122, Australia
}

\begin{abstract}
With the increase of complexity of modern software, social collaborative coding and reuse of open source software packages become more and more popular, which thus greatly enhances the development efficiency and software quality. However, the explosive growth of open source software packages exposes developers to the challenge of information overload. While this can be addressed by conventional recommender systems, they usually do not consider particular constraints of social coding such as social influence among developers and dependency relations among software packages. In this paper, we aim to model the dynamic interests of developers with both social influence and dependency constraints, and propose the Session-based Social and Dependency-aware software Recommendation (SSDRec) model. This model integrates recurrent neural network (RNN) and graph attention network (GAT) into a unified framework. An RNN is employed to model the short-term dynamic interests of developers in each session and two GATs are utilized to capture social influence from friends and dependency constraints from dependent software packages, respectively. Extensive experiments are conducted on real-world datasets and the results demonstrate that our model significantly outperforms the competitive baselines. Keywords: Software recommendation, Social network, Dependency network, Graph neural network
\end{abstract}

\footnotetext{
${ }^{*}$ Corresponding author

Email addresses: yanzhou@ahu.edu.cn (Dengcheng Yan), tangtianyi202012@163.com (Tianyi Tang), xiewxahu@foxmail.com (Wenxin Xie), zhangyiwen@ahu.edu.cn (Yiwen Zhang), qhe@swin.edu.au (Qiang He)
} 


\section{Introduction}

Modern software is becoming more and more complicated and the development usually needs collaboration of a team and depends on a large number of third-party software packages, which promotes the wide adoption of social collaborative coding paradigm. Recently, social collaborative coding platforms such as GitHub have emerged to provide developers with abundant functionalities of social collaboration and technical development and produce a large amount of high-quality open source software packages. According to GitHub 17 there are more than 56 million developers collaborating on more than 100 million software projects as of March, 2021.

While the explosive growth of open source software packages will significantly fuel the prosperity of the software industry, it also exposes developers to the challenge of information overload. Developers often need to spend much time searching software packages they are interested in. To address this challenge, it is essential to introduce recommendation systems which have been proven powerful to deal with information overload problem in various fields [1, 2, 3, 4, 5].

Recently, conventional recommendation models have been applied to software recommendation [6, 7, 8], but they usually do not consider either the dynamics of developers' interests [9] or particular constraints of social coding such as social influence among developers and dependency relations among software packages.

Actually, in the community of software development, developers' interests are dynamic and gradually evolve due to the emergence of new project requirements or new techniques. At different time periods, a developer may focus on a different technical field and is interested in software packages related to that technical field. Taking Derek Murray (GitHub ID: mrry), a main contributor of the popular deep learning framework TensorFlow, as an example, we can find from Figure 1 that he began to contribute to TensorFlow upon its establishment in GitHub while his contribution stopped in the middle of the year 2020. From his profile page in GitHub (shown in Figure 2), we can find that in the middle of the year 2020 his interest evolves to ONNX, an open standard for machine learning interoperability, and its

\footnotetext{
${ }^{1}$ https://github.com/about
} 
related projects. Thus, it is practical to capture developers' dynamic interests in the scenario of software recommendation. Practically, the dynamic interest of each developer can be captured from the projects he/she recently starred or contributed by modeling the sequence of these projects with recurrent neural network (RNN). For example, for each month, we group the projects Derek Murray starred or contributed in time order and the embedding of each project (obtaining from the output of the GAT on dependency network) is then input to an RNN one by one. After all projects are finished, the final output of the RNN is obtained as the representation of Derek Murray's interest in this time period.

Nov 1, 2015 - Oct 18, 2021

Contributions: Commits -

Contributions to master, excluding merge commits and bot accounts
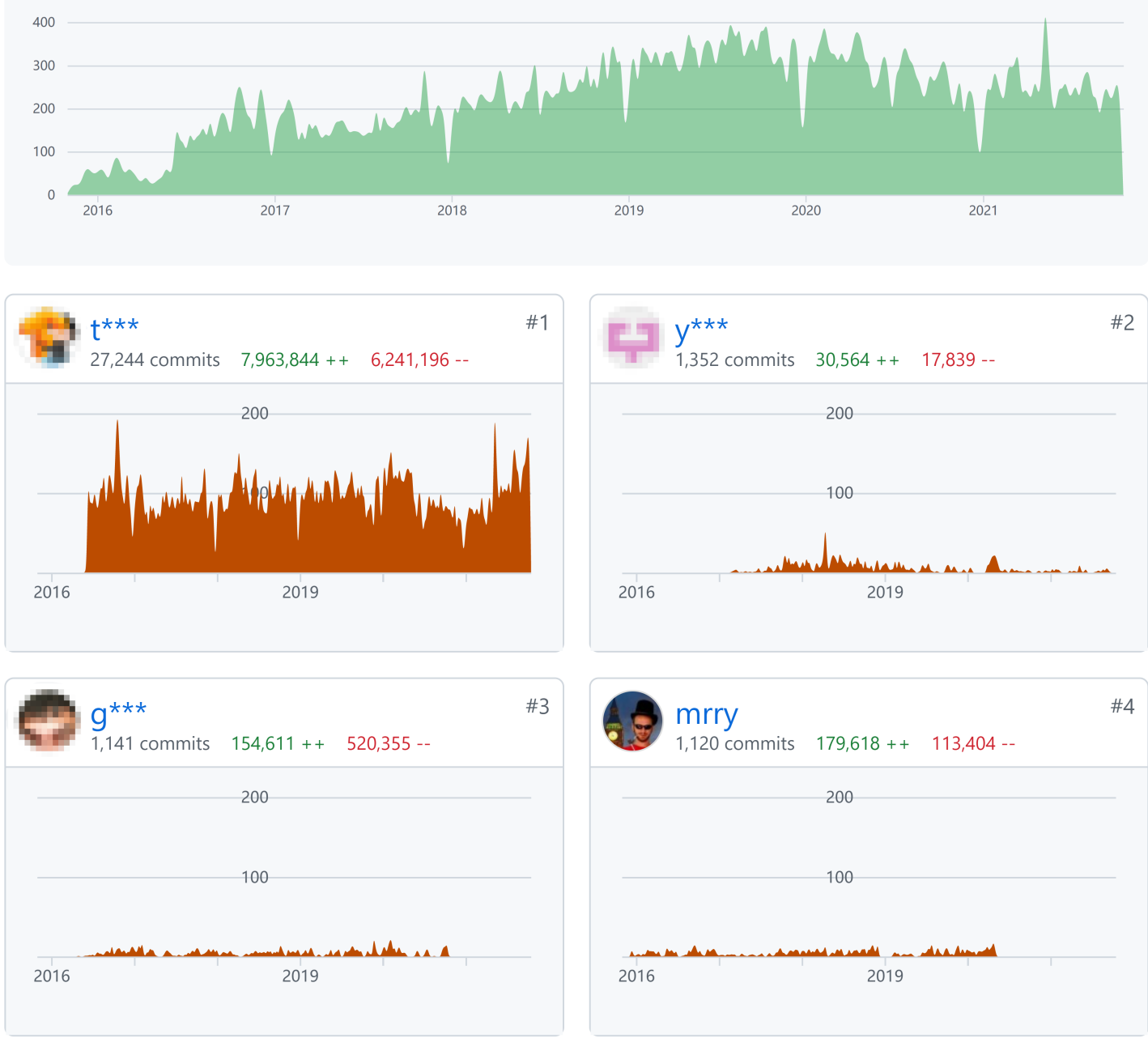

Figure 1: Contributors of project TensorFlow as of Oct. 18, 2021 2 


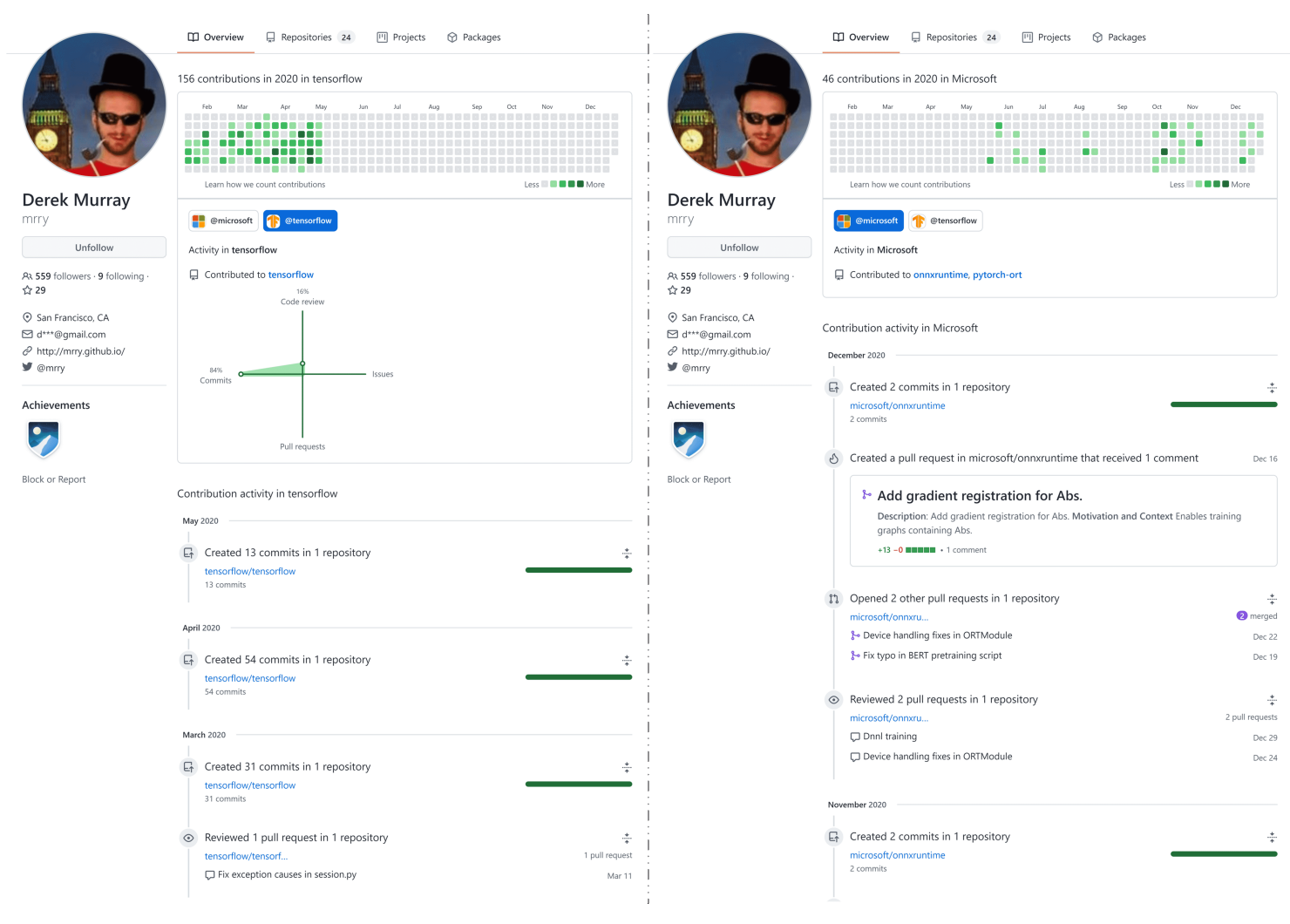

Figure 2: Dynamic interest of Derek Murray $]^{3}$

Recently, as social collaborative coding is becoming a popular paradigm for software development, social collaborative coding platforms such as GitHub emerge and provide abundant social functionalities such as forming social relations with other developers in addition to plentiful software development tools. A developer in GitHub can follow other developers as friends and obtain in his/her timeline their recent behaviors/choices such as starring or creating a new repository. Then, the developer's choice on software packages will be influenced by his/her friend developers' choices because of social trust and time efficiency as there are large amount of software projects in GitHub, it is efficient to make choice based on friend developers' choice. For example, Figure 3 shows the GitHub timeline of one of the authors Dengcheng Yan. The recent behaviors of the developers Dengcheng Yan has followed appears in his GitHub timeline. As a developer, Dengcheng Yan frequently reads his GitHub timeline and he found developer $\mathrm{r}^{* * *}$ starred an interesting project norvigpytudes.

\footnotetext{
${ }^{2}$ Obtained from https://github.com/tensorflow/tensorflow/graphs/contributors with privacy information hidden.

${ }^{3}$ Obtained from https://github.com/mrry with the consent of Derek Murray.
} 
Then he also starred this project. This phenomenon of social influence widely exists in GitHub and forms the intuition behind our proposal to employ social influence to enhance software recommendation. However, social relationship may have bad effect when a developer shift from one program language to another. So we should not treat all social relations equally. Actually, different social relations are weighted differently during different time periods. Specifically, we employ a graph attention network (GAT) on the social network and calculate the attention coefficient (i.e., strength of social influence) between developers by ingesting not only the social network structure but also the dynamic interest of developers. The more similar the dynamic interest between developers is, the stronger the social influence between them will be. In different time period, the dynamic interest of developers captures their recent preference and is different, thus making the strength of social influence adaptive to avoid outdated social relations.

Last and most importantly, software recommendation is different from conventional recommendation scenarios and dependency exists naturally between items (i.e., software packages) as a newly developed software package usually reuses the functionality of multiple existing software packages. Developers who develop the newly developed software packages should first be familiar with some of the functionalities of the dependency software packages. Thus, dependency constraints are another key factor that influences developers' choices which is essential to be considered in the software recommendation scenario.

We give a real-world motivation example which combines dynamic interest, social influence and dependency constraints of the popular python-based deep learning technical stacks, including both TensorFlow-based and PyTorch-based technical stacks(see Figure 4). In the first session, developer A has chosen DeepCell-tf (DCTF, for cell image analysis) and DeepCell-RetinaMask (DCRM, for cell object detection), we could conclude from both social influence and dependency constraints that he/she mainly focuses on developing tools for cell image analysis and would further extend his/her interest from simply detecting cells to tracking cells for cell lineage con-

\footnotetext{
${ }^{4}$ Obtained from https://github.com with privacy information hidden.
} 
All activity

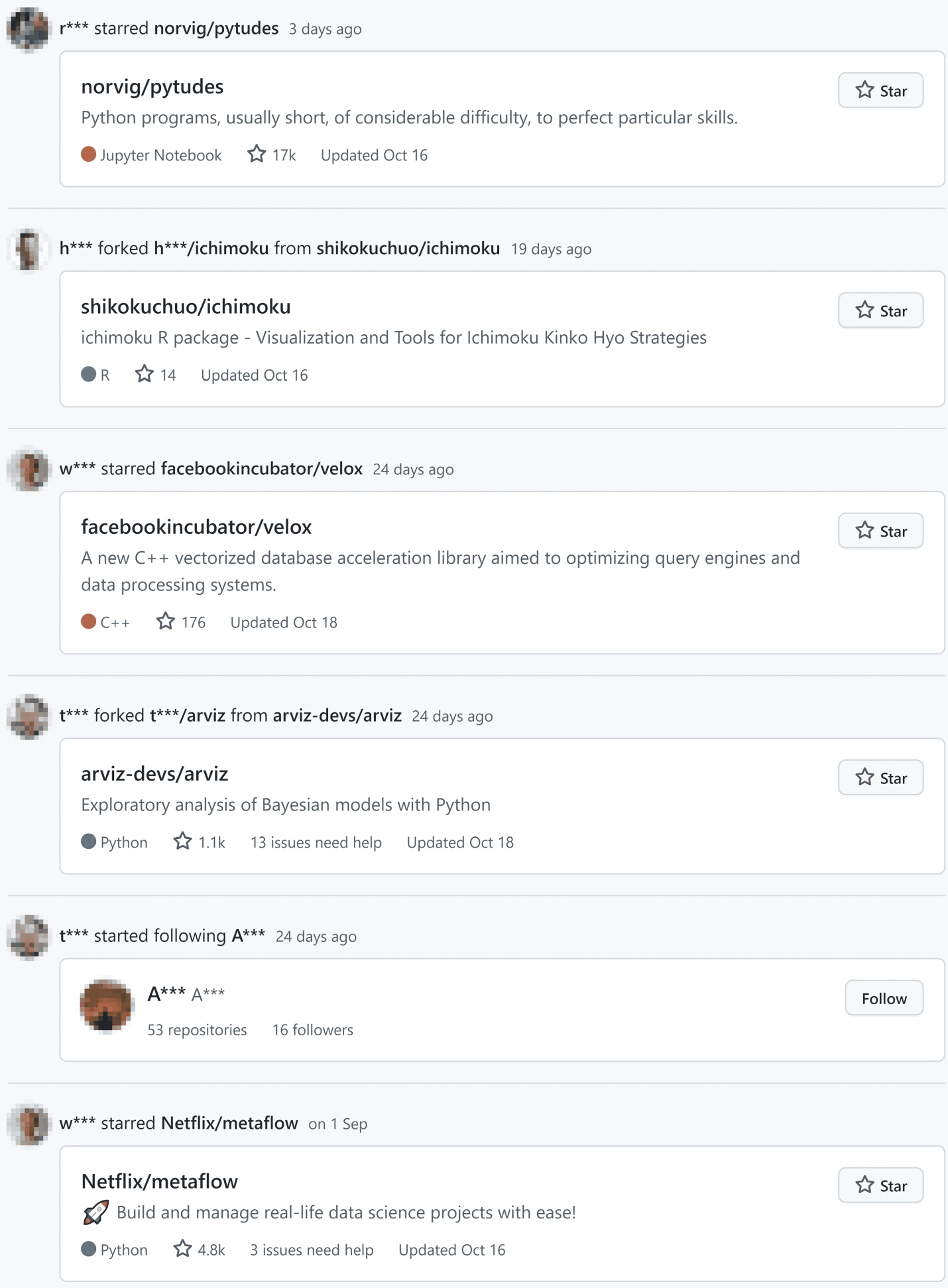

Figure 3: GitHub timeline of the author Dengcheng Yan $4^{4}$ 
struction using DeepCell-Tracking (DCT). Then after some period, in the second session, developer A would further analyze the cell lineage he/she has constructed but finds no suitable tools in TensorFlow-based technical stack. He/she turns to PyTorch-based technical stack and pays more attention to developers who have chosen PyTorch-related software packages. Finally, he/she chooses PyTorch (Torch) and PyTorch-Geomertic (TG, basically provides graph and tree analysis functions which are suitable for cell lineage analysis), and will further choose Torch-Cluster (TC) for cell lineage cluster analysis.

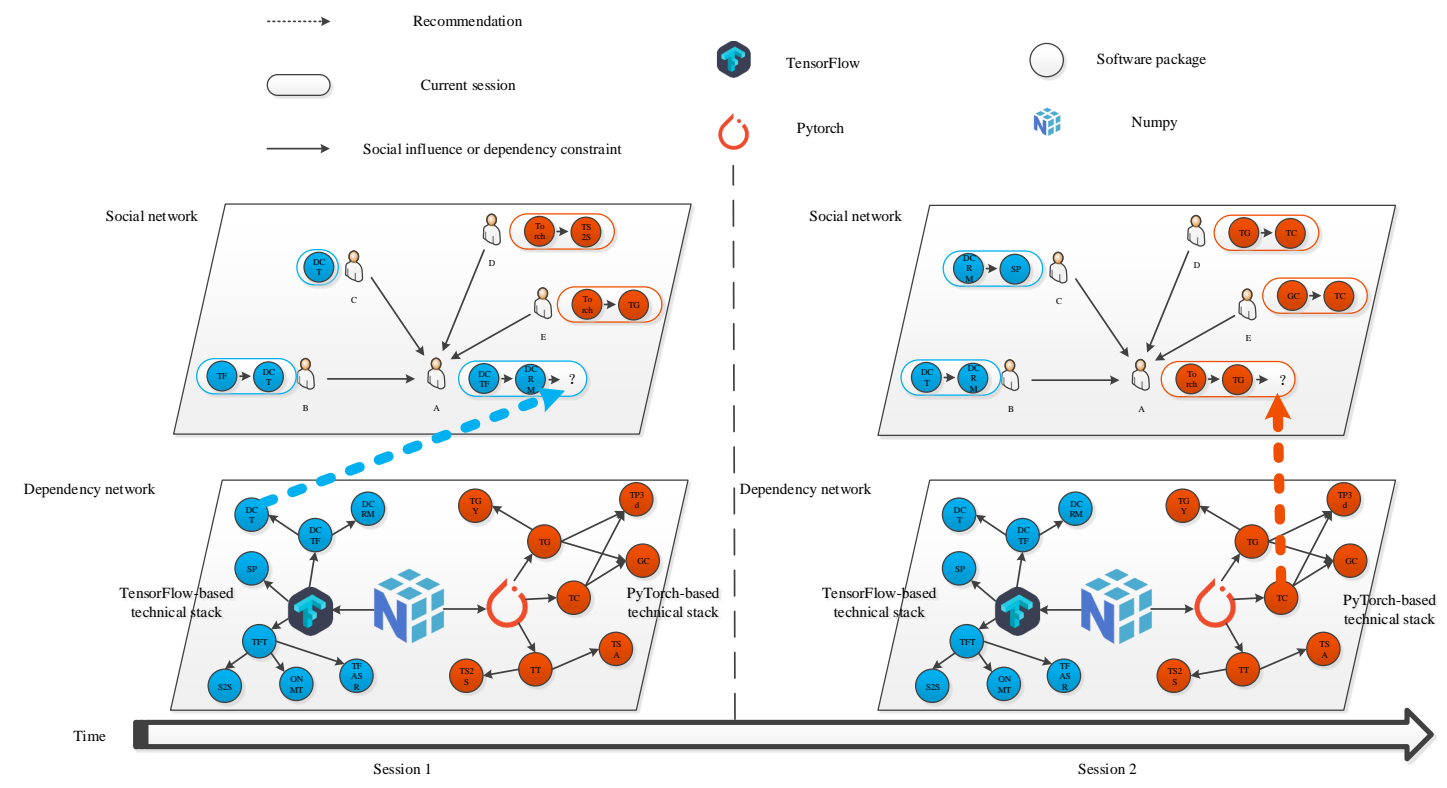

Figure 4: Motivation example. Due to space constraints, we utilize the abbreviations of the software packages to represent them and their full names are listed in table 1.

In this article, we focus on modeling the dynamic interests of developers with both social influence and dependency constraints, and propose the Session-based Social and Dependency-aware software Recommendation (SSDRec) model. Our main contributions are summarized as follows:

- We propose to model the dynamic interests of developers with both social influence from friends and dependency constraints among software packages.

- We develop a unified framework to integrate a recurrent neural network and two graph attention networks. The recurrent neural network models the short- 
Table 1: The abbreviations and full names of software packages.

\begin{tabular}{cc|cc}
\hline \multicolumn{2}{c|}{ TensorFlow } & \multicolumn{2}{c}{ PyTorch } \\
\hline Abbreviation & Full Name & Abbreviation & Full Name \\
\hline TF & TensorFlow & Torch & PyTorch \\
DCTF & DeepCell-tf & TG & Torch-Geometric \\
DCT & DeepCell-Tracking & TC & Torch-Cluster \\
DCRM & DeepCell-RetinaMask & GC & GraphChem \\
SP & Spektral & TP3d & Torch-Points3d \\
TFT & TensorFlow-Text & TT & Torch-Text \\
S2S & Seq2Seq & TS2S & PyTorch-Seq2Seq \\
TFASR & TensorFlowASR & TSA & PyTorch-Sentiment-Analysis \\
ONMT & OpenNMT & TGY & PyTorch-Geometric-YooChoose \\
\hline
\end{tabular}

term dynamic interests of developers in each session and the two graph attention networks capture social influence from friends and dependency constraints from dependent software packages, respectively.

- We evaluate the proposed model on three real-world datasets from different communities in GitHub, including PHP, Ruby and JavaScript. The results demonstrate the performance and effectiveness of our proposed model compared with the baselines.

The rest of this article is organized as follows. In Section 2, we briefly review the important work related to this paper. Section 3 provides some preliminaries of the article. Our proposed model SSDRec is described in Section 4 and the experiment results are shown in Section 5. Finally, we draw conclusions in Section 6 .

\section{Related work}

In this section, we will briefly review the related works in dynamic recommendation, social recommendation and software recommendation. 


\subsection{Dynamic recommendation}

As users' interests dynamically evolve, conventional recommendation models which capture users' long-term static interests are not applicable anymore and various dynamic recommendation models have been proposed. For example, an earlier work utilized Markov Chains in successive point-of-interest recommendation [10]. With the success of RNN in several domains such as stock market prediction [1] and sentiment analysis [12, various approaches [13, 14] have been proposed to exploit Recurrent Neural Networks (RNN) to model users' dynamic interests from their recent behaviors. Manotumruksa et al. [13] integrated the sequential time information of users' behaviors into the matrix factorization (MF) with an RNN. Dong et al. 14 further performed joint optimization of RNN and MF with shared parameters in a multi-task learning framework.

Furthermore, instead of modeling behavior sequence as a whole as the above models, session-based recommendation models segment users' behavior sequence into several sessions to model users' dynamic interests in a more fine-grained granularity. Hidasi et al. [15] first proposed an RNN-based approach for session-based recommendations to capture users' short-term dynamic interests within a session. Then based on the assumption that a session often serves different purposes, Wang et al. [16] proposed a mixture-channel model with attention mechanism to detect the purposes of each item. In addition to modeling interactions within a session, information across sessions has been also introduced. Ruocco et al. [17] exploited two separate RNNs to process the current session and the past sessions separately.

\subsection{Social recommendation}

Social recommendation utilizes social network information to enhance the performance of recommendation models. Conventionally, this information is utilized by some hand-crafted features. Ma et al. [18] regularized the matrix factorization framework with social network information. Zhao et al. [19] leveraged friends' interaction as another positive feedback for Bayesian Personalized Ranking (BPR). Ding et al. 20] combined social information and crow computing into factorization machines for recommendation. 
Recently, deep neural network is adopted to process the social network information in recommendation models instead of hand-crafted features. Deng et al. [21] utilized Autoencoder to initialize vectors in MF and updated them with both social trust ensemble and community effect. In order to extract structure information of social network more efficiently, graph neural network (GNN) is applied to recommendation systems [22]. Specifically, graph attention network [23] is usually utilized in recommendation systems to aggregate information from neighbors in a social network adaptively by learning different weights for different neighbors, which is also verified in rumor detection in social media [24]. Fan et al. [25] coherently modeled two graphs and heterogeneous strengths to jointly capture interactions and opinions in the user-item graph. Song et al. [26] further considered the evolution of social influence and proposed DGRec to jointly utilize RNN and GAT to capture distinguishing social influence from different friends in different time period. Inspired by the idea behind DGRec and other social recommendation models of utilizing social influence to improve recommendation performance, we further extend this idea to a more generalized form. We consider the interdependent relations among not only users (i.e., social network) but also items (i.e., software dependency network), and propose a unified framework to extract information from both of these two interdependent relations in an end-to-end manner. Specifically, we apply our model to the field of software engineering where the items (i.e., software packages) are naturally interdependent and the interdependent relations are a key factor to affects developers' choices on software packages.

\subsection{Software recommendation}

Software recommendation emerges as a hot research field with the rapid development of social collaborative coding platforms such as GitHub and explosive growth of third-party software packages. Conventional software recommendation usually employed collaborative filtering-based recommendation models [1, 2]. For example, Ichii et al. 6] used collaborative filtering to recommend similar software packages to developers. Thung et al. [7] further combined association rule and collaborative filtering to capture deeper relationships between software packages. He et al. [8] 
employed an adaptive weighting mechanism and neighborhood information to neutralize popularity bias in MF, which significantly increased both the diversity and accuracy of the recommendation results. In addition to directly reusing third-party software packages, Web service API is also reusable and recommendation models have been developed [27, 28].

As social collaborative coding becomes popular and development stages change rapidly, some software recommendation models have begun to employ social influence or developers' dynamic behaviors. Guy et al. [29] aggregated developers' familiarity network and similarity network to recommend social software. Jiang et al. [9] adopted time decay factor and operation behaviors weight to model developers' dynamic interests in a popular programming platform, Scratch. However, social influence or developers' dynamic behaviors are usually modeled separately and the dependency relations among software packages are usually ignored in existing works.

\section{Preliminaries}

In this section, we will first introduce some necessary definitions and formulate the problem. The main notations are summarized in Table 2. Generally, sets, vectors and matrices are denoted as upper-case letters, bold lower-case letters and bold upper-case letters, respectively.

Definition 1 (Session). An ordered set of software packages with which the developer has interacted within a specific time period. Let $U$ and I denote the sets of developers and software packages, respectively. The session of developer $u$ during time period $T$ is the ordered set of software packages developer $u$ watched within time period T, i.e., $S_{T}^{u}=<i_{T, 1}^{u}, i_{T, 2}^{u}, \cdots, i_{T, n}^{u}>\left(u \in U, i_{T, k}^{u} \in I\right)$.

In the software development community, developers need to keep investigating new software packages as new development requirements arise or new techniques emerge. Especially, developers pay attention to different technical fields during different time periods. Thus, developers' historical interactions with software packages need to be segmented into different groups by time periods to represent their different interests in a fine-grained granularity and to capture the evolution of the interests. 
Table 2: Summary of main notations

\begin{tabular}{c|l}
\hline Symbol & Description \\
\hline$U$ & The set of developers \\
\hline$G_{D}, G_{S}$ & The set of software packages \\
$S_{T}^{u}$ & The dependency network and the social network \\
$N_{D}(i)$ & $i$ 's one-hop neighbors in dependency network \\
$N_{S}(u)$ & $u$ 's one-hop neighbors in social network \\
$\alpha_{i j}^{(l)}$ & The attention weight between software package $i$ and $j$ in the $l$-th layer \\
$\beta_{g f}^{(l)}$ & The attention weight between developer $g$ and $f$ in the $l$-th layer \\
$\mathbf{h}_{t}$ & The hidden representation of LSTM at step $t$ \\
$\mathbf{e}_{i}^{(l)}, \mathbf{s}_{u}^{(l)}$ & the embeddings of software package $i$ and developer $u$ in $l$-th layer \\
$\mathbf{e}_{i}, \mathbf{s}_{u, T}$ & the final embeddings of software package $i$ and developer $u$ \\
$\mathbf{W}_{D}^{(l)}, \mathbf{W}_{S}^{(l)}$ & aggregation weight matrices for dependency and social network in $l$-th layer \\
$\mathbf{W}_{T D}, \mathbf{W}_{T S}$ & transformation matrices for dependency and social network \\
$p\left(i \mid S_{T}^{u}\right)$ & The probability that developer $u$ chooses package $i$ in condition of session $S_{T}^{u}$ \\
\hline
\end{tabular}


Definition 2 (Dependency constraint). The dependency network $G_{D}=\left(I, E_{D}\right)$ formed by the dependency relations among software packages constrains developer $u$ 's future choices to the near neighbors of his/her previous chosen software packages $\bigcup_{k=1}^{n} N_{D}\left(i_{T, k}^{u}\right)$.

As the complexity of modern software grows, it becomes a popular paradigm in modern software engineering to reuse third-party software packages. For example, to enable OAuth login using accounts from third-party social network platforms such as Facebook and Twitter, it is better to reuse well-tested third-party OAuth packages such as Laravel Socialite 5 , which will not only improve the efficiency of development but also guarantee the stability and security. Thus complicated dependency relations appear and form the dependency network. These dependency relations often constrain the technical choices of developers because developers often focus on specific technical fields which are in fact some sub-networks of the whole dependency network.

Definition 3 (Social influence). The choices of developer $u$ 's friend developers $N_{S}(u)$ in the developer social network $G_{S}=\left(U, E_{S}\right)$ influence u's future choices.

Similar to the dependency relations among software packages, developers in social collaborative coding platforms like GitHub usually follow other developers and build the social network $G_{S}$. Then the friend developers' activities such as watching or forking a new software project appear in the developer's timeline, which will influence the future technical choices of developers.

Problem 1 (Session-based software recommendation). Given a new session $S_{T}^{u}=<i_{T, 1}^{u}, \cdots, i_{T, n}^{u}>$ of developer $u$, the goal of session-based software recommendation is to recommend a set of software packages from $I$ that the developer is most likely to watch in the next step $n+1$ within session $T$, i.e., $i_{T, n+1}^{u}$.

In this article, we focus on the session-based software recommendation by simultaneously considering both dependency constraint among software packages and social influence among developers.

\footnotetext{
${ }^{5}$ https://github.com/laravel/socialite
} 


\section{Methodology}

In this section, we present the proposed Session-based Social and Dependencyaware software Recommendation model SSDRec in detail 6, The overview of the model is shown in Figure 5 and it is composed of four components: (1) Dependency constraint: we use a graph attention network to capture the dependency relations among software packages and obtain the embedding of each software package. (2) Dynamic interest modeling: we use a recurrent neural network to model the sequence of software packages in a session and obtain the final embedding of developer's dynamic interests in this session. (3) Social influence: we use another graph attention network to capture the social influence from neighbor developers and obtain the final embedding of each developer. (4) Recommendation: we use softmax to estimate the probability a developer will choose a given software package.

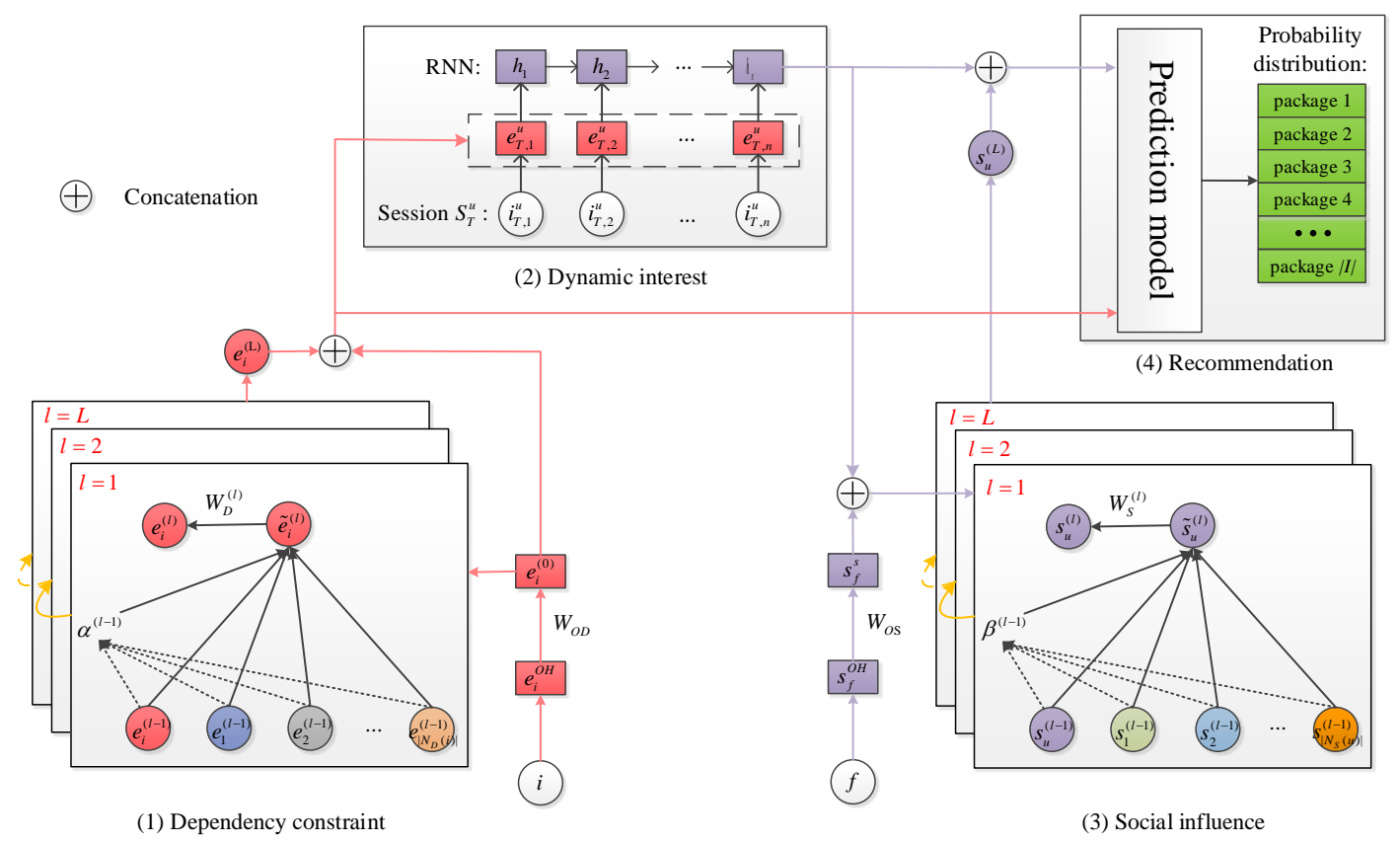

Figure 5: An overview of SSDRec.

\subsection{Dependency constraint}

Dependency constraint can be regarded as the domain knowledge specific for software recommendation. We employ this domain knowledge into the embeddings

\footnotetext{
${ }^{6}$ The code and data are available at https://github.com/SSOTTY/SSDRec
} 
of software packages by a graph attention network.

First, the initial embedding of each software package is obtained by a transformation of its one-hot encoding,

$$
\mathbf{e}_{i}^{(0)}=\mathbf{W}_{O D} \cdot \mathbf{e}_{i}^{O H}
$$

where $\mathbf{e}_{i}^{O H} \in \mathbb{R}^{|I|}$ and $\mathbf{e}_{i}^{(0)} \in \mathbb{R}^{E}$ are the software package $i$ 's one-hot encoding and initial embedding, respectively. $\mathbf{W}_{O D} \in \mathbb{R}^{E \times|I|}$ is the transformation matrix.

Then the attention mechanism is utilized to distinguish the different dependency constraints from different neighbor software packages and the attention weight between software package $i$ and $j\left(j \in N_{D}(i) \cup\{i\}\right)$ in the $l$-th layer of the graph attention network is calculated as

$$
\alpha_{i j}^{(l)}=\frac{\exp \left(\left(\mathbf{e}_{i}^{(l)}\right)^{T} \cdot \mathbf{e}_{j}^{(l)}\right)}{\sum_{k \in N_{D}(i) \cup\{i\}} \exp \left(\left(\mathbf{e}_{i}^{(l)}\right)^{T} \cdot \mathbf{e}_{k}^{(l)}\right)}
$$

where $\alpha_{i j}^{(l)} \in \mathbb{R}$ is the attention weight between software package $i$ and $j$, and $N_{D}(i)$ is the set of one-hop neighbors of software package $i$ in the dependency network. Note that we also use self-connection edge to preserve the embedding of software package $i$ itself.

Finally, the embedding of software package $i$ is updated by a weighed aggregation of the embeddings of its neighbor packages from the previous layer,

$$
\mathbf{e}_{i}^{(l+1)}=\sigma\left(\mathbf{W}_{D}^{(l+1)} \sum_{j \in N_{D}(i) \cup\{i\}} \alpha_{i j}^{(l)} \mathbf{e}_{j}^{(l)}\right)
$$

where $\mathbf{W}_{D}^{(l+1)} \in \mathbb{R}^{E \times E}$ is the aggregation weight matrix of the $(l+1)$-th layer and $\sigma$ is the nonlinear activation function.

After $L$ layers' aggregation of dependency constraints, the embedding $\mathbf{e}_{i}^{(L)}$ is obtained. We then concatenate it with its original embedding $\mathbf{e}_{i}^{(0)}$ as the final embedding of the software package,

$$
\mathbf{e}_{i}=\mathbf{W}_{T D} \cdot\left(\mathbf{e}_{i}^{(0)} \| \mathbf{e}_{i}^{(L)}\right)
$$

where $W_{T D} \in \mathbb{R}^{E \times 2 E}$ is a linear transformation matrix. 
The overall procedure is summarized in Algorithm 1 .

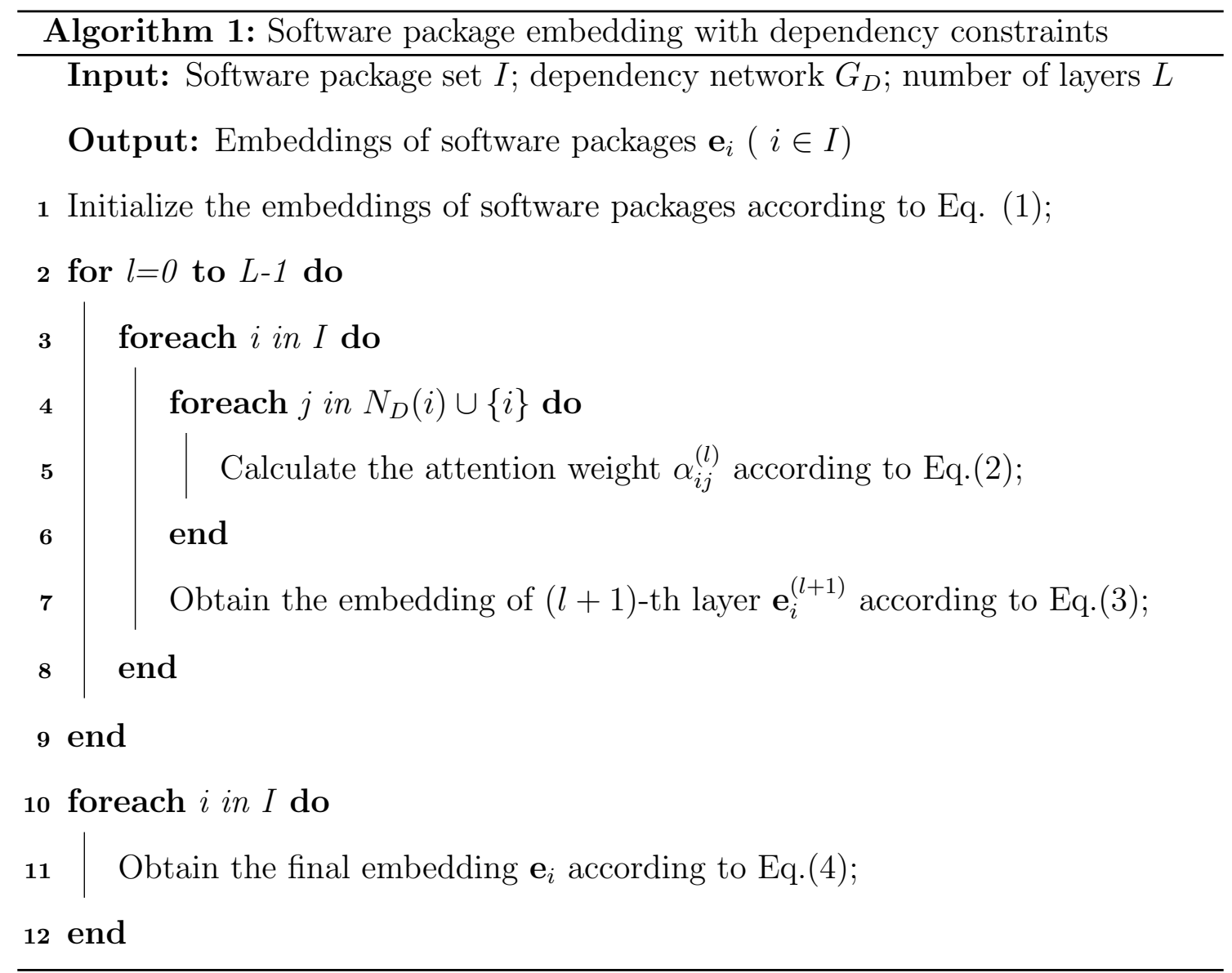

\subsection{Dynamic interest}

Developers' interests gradually evolve as development requirements change and new technology emerges. We capture developers' dynamic interests in each session by modeling the sequence of software packages within each session using a recurrent neural network (RNN).

Given the session $S_{T}^{u}=<i_{T, 1}^{u}, i_{T, 2}^{u}, i_{T, 3}^{u}, \ldots, i_{T, n}^{u}>$ of developer $u$ in time period $T$, the embeddings of the software packages in this session $<e_{T, 1}^{u}, e_{T, 2}^{u}, e_{T, 3}^{u}, \ldots, e_{T, n}^{u}>$ are first obtained from the previous step and then fed to an RNN. The RNN recurrently learns a hidden representation from the sequence by taking account both the current input package and previous input packages,

$$
\mathbf{h}_{t}=R N N\left(\mathbf{e}_{T, t}^{u}, \mathbf{h}_{t-1}\right)
$$

where $R N N$ is a kind of recurrent neural network model and $\mathbf{h}_{t} \in \mathbb{R}^{E}$ is the hidden representation with dimension $E$ at step $t$. 
Generally, there are various RNN models and we choose the long short-term memory (LSTM) model. Details of LSTM are shown in Equation (6),

$$
\begin{aligned}
\mathbf{f}_{t} & =\sigma\left(\mathbf{W}^{f} \mathbf{e}_{T, t}^{u}+\mathbf{U}^{f} \mathbf{h}_{t-1}+\mathbf{b}_{f}\right) \\
\mathbf{i}_{t} & =\sigma\left(\mathbf{W}^{i} \mathbf{e}_{T, t}^{u}+\mathbf{U}^{i} \mathbf{h}_{t-1}+\mathbf{b}_{i}\right) \\
\mathbf{o}_{t} & =\sigma\left(\mathbf{W}^{o} \mathbf{e}_{T, t}^{u}+\mathbf{U}^{o} \mathbf{h}_{t-1}+\mathbf{b}_{o}\right) \\
\hat{\mathbf{c}}_{t} & =\tanh \left(\mathbf{W}^{c} \mathbf{e}_{T, t}^{u}+\mathbf{U}^{c} \mathbf{h}_{t-1}+\mathbf{b}_{c}\right) \\
\mathbf{c}_{t} & =\mathbf{f}_{t} \odot \mathbf{c}_{t-1}+\hat{\mathbf{c}}_{t} \odot \mathbf{i}_{t} \\
\mathbf{h}_{t} & =\mathbf{o}_{t} \odot \tanh \left(\mathbf{c}_{t}\right)
\end{aligned}
$$

where $\mathbf{W} \in \mathbb{R}^{E \times E}, \mathbf{U} \in \mathbb{R}^{E \times E}$ and $\mathbf{b} \in \mathbb{R}^{E}$ are all model parameters, and $\odot$ denotes element-wise product.

The overall procedure is summarized in Algorithm 2 .

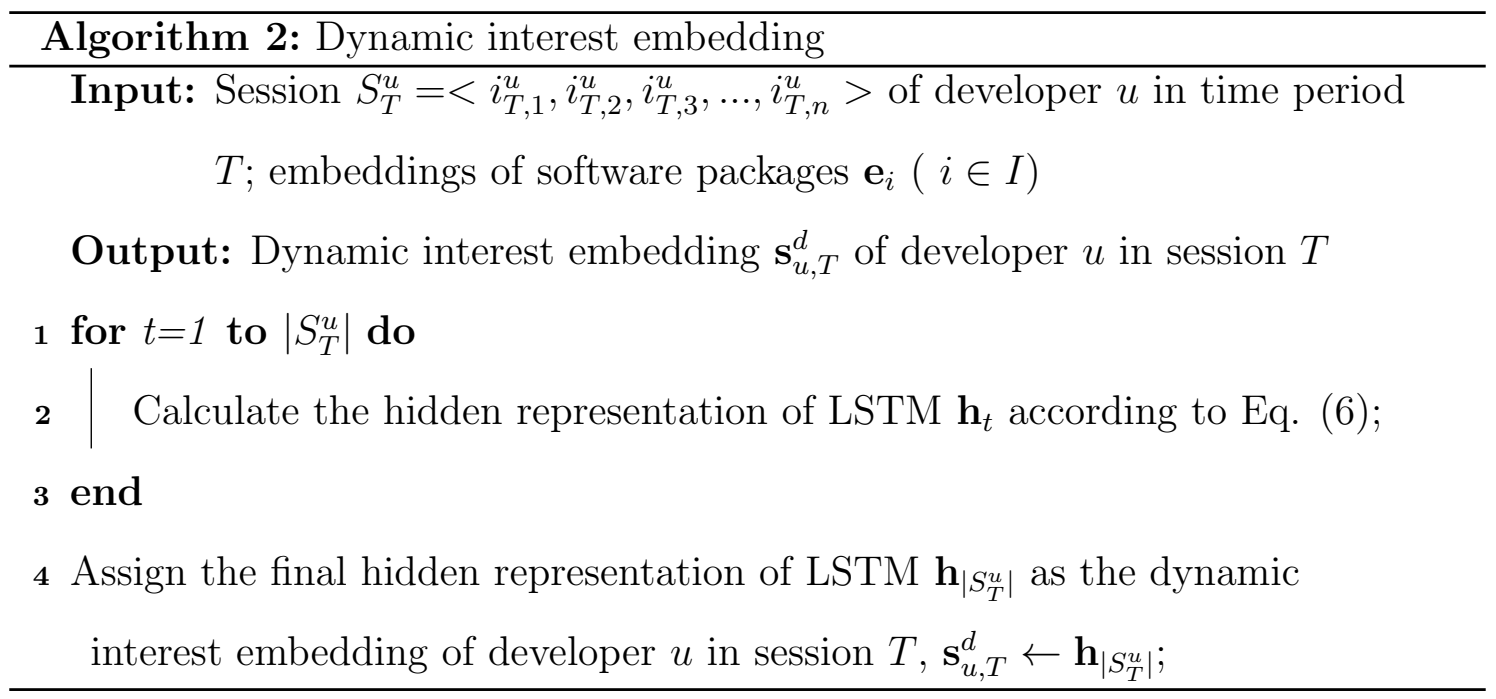

\subsection{Social influence}

In social collaborative coding platforms like GitHub, a developer can follow other developers to have their recent activities appeared in his/her timeline. Thus, neighbor developers' choices can influence the developer's own choice. We capture this social influence by applying a graph attention network on the social network of developers.

First, for a given developer $u$ at time period $T$, the initial embedding of each of his/her neighbor $f \in N_{S}(u)$ is obtained by a combination of his/her one-hot encoding and dynamic interest of previous time period $T-1$,

$$
\mathbf{s}_{f}^{(0)}=\sigma\left(\mathbf{W}_{f}\left(\mathbf{s}_{f, T-1}^{d} \| \mathbf{W}_{O S} \cdot \mathbf{s}_{f}^{O H}\right)\right)
$$


where $\mathbf{s}_{f}^{O H} \in \mathbb{R}^{|U|}, \mathbf{s}_{f, T-1}^{d} \in \mathbb{R}^{E}$ and $\mathbf{s}_{f}^{(0)} \in \mathbb{R}^{E}$ are the developer f's one-hot encoding, dynamic interest embedding at time period $T-1$ and initial embedding, respectively. $\mathbf{W}_{f} \in \mathbb{R}^{E \times 2 E}$ and $\mathbf{W}_{O S} \in \mathbb{R}^{E \times|U|}$ are the transformation matrices. The reason why neighbors' dynamic interest of previous time period is utilized comes from the practical situation that a session has a certain length (e.g., one week) while within a certain session $T$, developers' activities usually happen at a time point in a finer granularity (e.g., day). For example, in session $T$, the last activity of developer $u$ happens on Wednesday and to predict his/her preference in the following days in this session, it not reasonable to involve his/her neighbor $f$ 's activity which will happen in the future, e.g., Friday, in the modeling of social influence. Thus, we model the social influence from $T=2$ with neighbors' dynamic interest embeddings from the last session $T-1(T-1 \geq 1)$.

Specially, for the target developer $u$, we initialize his/her embedding using the dynamic interest embedding at time period $T$,

$$
\mathbf{s}_{u}^{(0)}=\mathbf{s}_{u, T}^{d}
$$

Then similar to dependency constraints, the attention mechanism is also utilized to distinguish the different social influence from different neighbor developers. The attention weight between developer $g$ and $f\left(f \in N_{S}(g) \cup\{g\}\right)$ in the $l$-th layer of the graph attention network is calculated as

$$
\beta_{g f}^{(l)}=\frac{\exp \left(\left(\mathbf{s}_{g}^{(l)}\right)^{T} \cdot \mathbf{s}_{f}^{(l)}\right)}{\sum_{k \in N_{S}(g) \cup\{g\}} \exp \left(\left(\mathbf{s}_{g}^{(l)}\right)^{T} \cdot \mathbf{s}_{k}^{(l)}\right)}
$$

where $\beta_{g f}^{(l)} \in \mathbb{R}$ is the attention weight between developer $g$ and $f$, and $N_{S}(g)$ is the set of one-hop neighbors of developer $g$ in the social network.

Finally, the embedding of developer $g$ at time period $T$ is updated by a weighed aggregation of the embeddings of its neighbor developers from the previous layer,

$$
\mathbf{s}_{g}^{(l+1)}=\sigma\left(\mathbf{W}_{S}^{(l+1)} \sum_{f \in N_{S}(g) \cup\{g\}} \beta_{g f}^{(l)} \mathbf{s}_{f}^{(l)}\right)
$$

where $\mathbf{W}_{S}^{(l+1)} \in \mathbb{R}^{E \times E}$ is the aggregation weight matrix of the $(l+1)$-th layer and $\sigma$ is the nonlinear activation function. 
After $L$ layers' aggregation of social influence, the embedding $\mathbf{s}_{u}^{(L)}$ is obtained. We then concatenate it with its initial embedding $\mathbf{s}_{u}^{(0)}$ as the final embedding of developer $u$,

$$
\mathbf{s}_{u, T}=\mathbf{W}_{T S} \cdot\left(\mathbf{s}_{u}^{(0)} \| \mathbf{s}_{u}^{(L)}\right)
$$

where $W_{T S} \in \mathbb{R}^{E \times 2 E}$ is a linear transformation matrix.

The overall procedure is summarized in Algorithm 3 .

\begin{tabular}{l}
\hline Algorithm 3: Developer embedding with social influence \\
\hline Input: Target developer $u$; time period $T$; session set $S=\left\{S_{T}^{u}\right\}$; social \\
network $G_{S}$; neighborhood sampling functions $N_{S}^{(l)} ;$ number of \\
layers $L$
\end{tabular}

Output: Developer $u$ 's embedding $\mathbf{s}_{u, T}$

1 Initialize neighbor set $B^{L} \leftarrow\{u\}$;

2 for $k=L$ to 1 do

$3 \quad$ Initialize neighbor set $B^{k-1} \leftarrow B^{k}$;

$4 \quad$ foreach $g$ in $B^{k}$ do

$5 \quad$ Sample neighbors of $g$ using neighborhood sampling function $N_{S}^{(k)}(g)$ and add to neighbor set $B^{k-1} \leftarrow B^{k-1} \cup N_{S}^{(k)}(g)$;

6 end

7 end

8 foreach $g$ in $B^{0}$ do

$\mathbf{9}$ Obtain the initial embedding $\mathbf{s}_{g}^{(0)}$ of $g$ according to Eq. 77 and Eq. (8); 10 end

11 for $k=1$ to $L$ do

12 foreach $g$ in $B^{k}$ do

13 Calculate attention weight between $g$ and its neighbors according to Eq. (9); Calculate the embedding $\mathbf{s}_{g}^{(k)}$ of $g$ in $k$-th layer according to Eq. 10 ;

15 end

16 end

17 Calculate the final embedding $\mathbf{s}_{u, T}$ of developer $u$ according Eq. 11 ; 


\subsection{Recommendation}

After obtaining the embeddings of the software packages and the developer $u$, we employ the softmax to estimate the probability that developer $u$ will choose each software package $i$ and recommend the top- $K$ software packages to developer $u$.

$$
p\left(i \mid S_{T}^{u}\right)=\frac{\exp \left(\mathbf{e}_{i}^{T} \cdot \mathbf{s}_{u, T}\right)}{\sum_{z=1}^{|I|} \exp \left(\mathbf{e}_{z}^{T} \cdot \mathbf{s}_{u, T}\right)}
$$

\subsection{Optimization}

To learn the model parameters, we maximize the log-likelihood of the observed packages in all sessions,

$$
\sum_{u \in U} \sum_{t=2}^{T} \sum_{n=1}^{L_{u, t}-1} \log \left(p\left(i_{t, n+1}^{u} \mid i_{t, 1}^{u}, \ldots, i_{t, n}^{u}\right)\right)
$$

where $L_{u, t}$ denotes the lenghth of session $t$ of developer $u$. As our model is an end-to-end framework, all components (i.e., two GATs and an RNN) are combined into a single neural network model for optimization. In particular, we optimize the model parameters by utilizing the widely-adopted data augmentation and minibatch technique. Specifically, we first make the first $m$ items of a session as a new session and then combine every $M$ sessions into a batch for a round of parameter updating. 
The overall training procedure is summarized in Algorithm 4 .

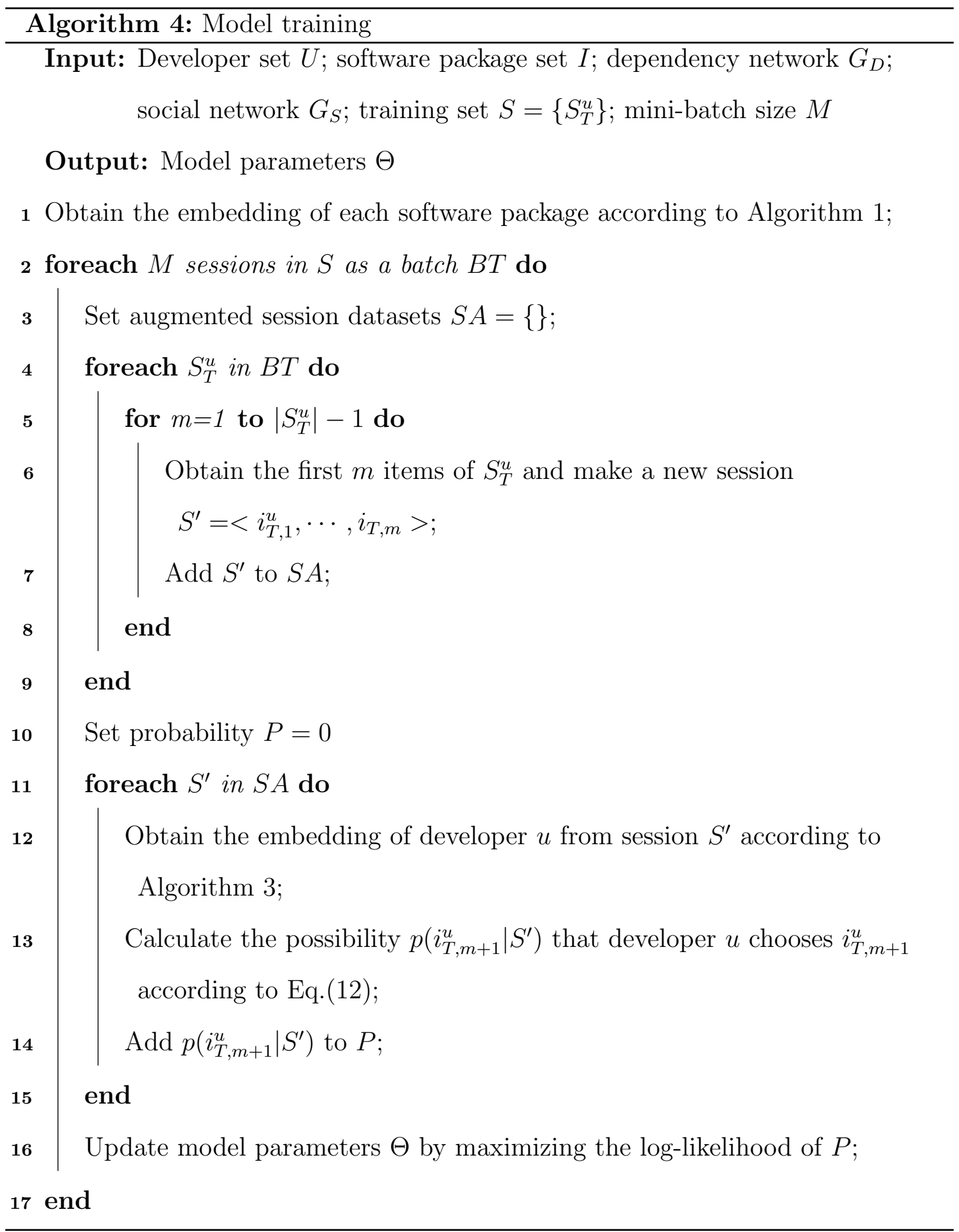

\section{Experiments}

In this section, we conduct experiments to answer the following research questions:

- RQ1 Does SSDRec outperform the baseline methods on all experimental settings? 
- RQ2 Do the components of SSDRec enhance the effectiveness by (a) modeling social influence and dependency constraints, (b) capturing friends' dynamic and static interests.

- RQ3 How do some hyper-parameters affect the performance of SSDRec?

In the remainder of this section, we will first describe the experimental settings (Section 5.1) and then answer the above research questions(Section 5.2, Section 5.3 . Section 5.4). Finally, several illustrative examples are given to verify our assumptions.(Section 5.5).

\subsection{Experimental Settings}

\subsubsection{Datasets}

To evaluate the proposed approach, we integrate the datasets of GHTorrent [30] and Libraries.io 7. GHTorrent monitors the public event timeline of Github and provides abundant social relations between developers and development interactions between developers and software repositories from the popular social collaborative coding platform GitHub. And Libraries.io provides the explicit dependency relations between software packages. In our experiments, we extract three datasets from the integration of GHTorrent and Libraries.io according to the programming language, i.e., PHP, Ruby and JavaScript. For each extracted dataset, the social relations between developers, the watch interactions between developers and software repositories, and the dependency relations between software packages are extracted. To ensure enough social awareness for each developer, we only extract those developers who follow more than $m$ developers and are followed by more than $n$ developers. Similarly, we keep the software packages that are watched by at least $k$ developers. Due to the different popularity of those three programming languages, we set $m=20,50,100, n=50,100,200$ and $k=20,20,50$ for PHP, Ruby and JavaScript, respectively. Then the watched items of each developer are divided into week-long sessions and only those sessions whose length is within $[2,30]$ are kept according to [26].

\footnotetext{
${ }^{7}$ https://libraries.io/data
} 
Table 3: The statistics of datasets.

\begin{tabular}{lccc}
\hline Dataset & PHP & Ruby & JavaScript \\
\hline \# Developers & 7,095 & 5,935 & 8,144 \\
\# Software packages & 1,402 & 1,487 & 2,282 \\
\# Interactions & 118,256 & 116,832 & 436,409 \\
\hline Avg. friends per developer & 8.59 & 13.05 & 16.24 \\
Avg. dependencies per package & 4.56 & 5.09 & 10.09 \\
Avg. session length & 2.95 & 3.13 & 3.25 \\
\hline
\end{tabular}

Each dataset is then split into training, validation and test sets. Sessions of each developer are ordered by time in ascending order and the sessions within the last two years (about 104 weeks) are randomly split into validation and test sets. The rest sessions are used as training set. Note that when splitting datasets, we ensure all packages in validation/test sets appear in the training set. The detailed statistics of these three datasets are shown in Table 3 .

\subsubsection{Baselines}

To evaluate the performance of our proposed SSDRec, we compare it with the following baselines. As software recommendation can utilize conventional recommendation models with specific features, we choose the two classic recommendation modes, i.e., BPR [1] and NCF [2] as baselines. Moreover, considering the dynamic nature we also choose two session-based recommendation models, i.e., RNN [15] and DGRec [26]. As for recommendation models specifically designed for software recommendation, because they usually focuses on enhancing recommendation performance by incorporating additional meta information, such as tag and domainspecific knowledge [31], programming language [32], description files [33], textual requirement descriptions [34] and multiple developer-software interactions [35] and there is hardly any software recommendation models utilizing the natural dependency relations among software packages except RepoLike [36]. We also choose RepoLike as a baseline. A brief introduction of these baselines are depicted as follows: 
- BPR [1]: a classical MF-based method optimized with a ranking objective.

- NCF [2]: uses neural network instead of inner product to model relationship between users and items of MF.

- ReopLike [36]:a personalized recommendation approach that uses project popularity, technical dependencies and social connections for open-source repositories.

- RNN [15]: captures users' session-level dynamic interests with RNN.

- DGRec [26]: a state-of-the-art model for session-based social recommendation. It utilizes RNN and GAT to model dynamic interests and dynamic social influence.

\subsubsection{Parameter Settings}

Our proposed model is implemented using TensorFlow [37]. Adam [38] is chosen as optimizer and parameters are initialized as suggested in [37]. The batch size and dropout are set to 200 and 0.2 , respectively. The embedding dimensions of both developers and software packages for all models are set to 100. For both social network and dependency network, we employ the graph attention networks with 2 layers, i.e., $L=2$ according to [39] and for each node 10 one-hop neighbors and 5 two-hop neighbors are sampled as recommended in [39]. All experiments are run on a machine with a GeForce RTX2080Ti GPU.

\subsubsection{Evaluation Metrics}

The performance of SSDRec and all baselines are evaluated with two wellknown metrics: Hit Rate $H R @ K$ and Normalized Discounted Cumulative Gain $N D C G @ K$.

$H R @ K$ measures the proportion of users who get correct recommendation results and it can be formulated as

$$
H R @ K=\frac{\sum_{u \in U} I\left(R_{K}(u) \cap T(u)\right)}{|U|}
$$


where $R_{K}(u)$ and $T(u)$ are the sets of top-K recommendation list and ground truth list for user $u$, respectively. $I(\cdot)$ is the indicator function and $I\left(R_{K}(u) \cap T(u)\right)=0$ when $R_{K}(u) \cap T(u)=\emptyset$ otherwise it is 1 . - $\mathrm{U}$ - is the total number of users.

$N D C G @ K$ considers the orders of users' preferred items in the ranked Top$\mathrm{N}$ recommendation list for users usually pay attention to only the top few items recommended by a recommendation system. It can be formulated as:

$$
N D C G @ K=\frac{1}{|U|} \sum_{u \in U} \frac{1}{\log _{2}(1+\operatorname{pos}(T(u)))}
$$

where $\operatorname{pos}(T(u))$ is the order of the top ranked item of $u$ 's ground truth items in the recommendation list.

\subsection{Overall performance (RQ1)}

Table 4: The overall performance. The Improvement rate is calculated between the best and the $\underline{\text { second best performed models. }}$

\begin{tabular}{|c|c|c|c|c|c|c|c|}
\hline \multirow{2}{*}{ Dataset } & \multirow{2}{*}{ Model } & \multicolumn{3}{|c|}{$H R @ K(\%)$} & \multicolumn{3}{|c|}{$N D C G @ K(\%)$} \\
\hline & & 10 & 20 & 50 & 10 & 20 & 50 \\
\hline \multirow{6}{*}{ PHP } & $\mathrm{BPR}$ & $3.41 \pm 0.159$ & $6.70 \pm 0.104$ & $15.26 \pm 0.111$ & $1.40 \pm 0.064$ & $2.22 \pm 0.048$ & $3.90 \pm 0.050$ \\
\hline & $\mathrm{NCF}$ & $4.50 \pm 0.054$ & $8.13 \pm 0.040$ & $18.35 \pm 0.161$ & $2.08 \pm 0.025$ & $2.99 \pm 0.002$ & $5.00 \pm 0.031$ \\
\hline & RepoLike & $5.54 \pm 0.111$ & $8.65 \pm 0.034$ & $15.02 \pm 0.055$ & $2.76 \pm 0.228$ & $3.59 \pm 0.026$ & $4.86 \pm 0.092$ \\
\hline & $\mathrm{RNN}$ & $9.75 \pm 0.099$ & $16.23 \pm 0.132$ & $28.72 \pm 0.238$ & $4.55 \pm 0.045$ & $6.22 \pm 0.033$ & $8.76 \pm 0.047$ \\
\hline & DGRec & $11.07 \pm 0.040$ & $17.29 \pm 0.020$ & $29.64 \pm 0.069$ & $5.37 \pm 0.024$ & $7.04 \pm 0.025$ & $9.55 \pm 0.015$ \\
\hline & SSDRec & $11.95 \pm 0.081$ & $18.93 \pm 0.135$ & $31.13 \pm 0.077$ & $5.88 \pm 0.042$ & $7.74 \pm 0.020$ & $10.22 \pm 0.005$ \\
\hline & Improvement rate $(\%)$ & 7.97 & 9.44 & 5.02 & 9.49 & 9.96 & 6.98 \\
\hline \multirow{7}{*}{ Ruby } & $\mathrm{BPR}$ & $2.09 \pm 0.155$ & $4.13 \pm 0.200$ & $10.16 \pm 0.094$ & $0.88 \pm 0.086$ & $1.39 \pm 0.098$ & $2.56 \pm 0.037$ \\
\hline & $\mathrm{NCF}$ & $2.83 \pm 0.098$ & $5.03 \pm 0.099$ & $11.17 \pm 0.088$ & $1.34 \pm 0.041$ & $1.88 \pm 0.037$ & $3.09 \pm 0.029$ \\
\hline & RepoLike & $5.97 \pm 0.331$ & $10.15 \pm 0.063$ & $17.54 \pm 0.045$ & $3.11 \pm 0.201$ & $4.17 \pm 0.025$ & $5.69 \pm 0.074$ \\
\hline & RNN & $6.25 \pm 0.071$ & $10.07 \pm 0.038$ & $19.17 \pm 0.075$ & $3.18 \pm 0.052$ & $4.19 \pm 0.078$ & $5.98 \pm 0.069$ \\
\hline & DGRec & $7.00 \pm 0.027$ & $10.86 \pm 0.027$ & $20.73 \pm 0.058$ & $3.68 \pm 0.006$ & $4.70 \pm 0.001$ & $6.68 \pm 0.017$ \\
\hline & SSDRec & $7.63 \pm 0.169$ & $12.05 \pm 0.072$ & $21.98 \pm 0.115$ & $4.01 \pm 0.047$ & $5.19 \pm 0.039$ & $\mathbf{7 . 1 7} \pm \mathbf{0 . 0 4 5}$ \\
\hline & Improvement rate $(\%)$ & 9.08 & 10.96 & 6.02 & 8.93 & 10.40 & 7.43 \\
\hline \multirow{7}{*}{ JavaScript } & BPR & $1.92 \pm 0.057$ & $3.59 \pm 0.074$ & $7.74 \pm 0.160$ & $0.85 \pm 0.029$ & $1.27 \pm 0.025$ & $2.08 \pm 0.019$ \\
\hline & $\mathrm{NCF}$ & $2.32 \pm 0.012$ & $4.11 \pm 0.130$ & $8.42 \pm 0.107$ & $1.07 \pm 0.029$ & $1.52 \pm 0.058$ & $2.37 \pm 0.054$ \\
\hline & RepoLike & $2.50 \pm 0.029$ & $4.40 \pm 0.037$ & $8.53 \pm 0.039$ & $1.13 \pm 0.026$ & $1.64 \pm 0.017$ & $2.46 \pm 0.090$ \\
\hline & $\mathrm{RNN}$ & $4.59 \pm 0.021$ & $7.91 \pm 0.100$ & $15.27 \pm 0.102$ & $2.19 \pm 0.005$ & $3.06 \pm 0.029$ & $4.53 \pm 0.024$ \\
\hline & DGRec & $5.26 \pm 0.046$ & $8.87 \pm 0.061$ & $17.58 \pm 0.065$ & $2.53 \pm 0.005$ & $3.47 \pm 0.024$ & $5.22 \pm 0.031$ \\
\hline & SSDRec & $5.70 \pm 0.085$ & $9.73 \pm 0.149$ & $18.47 \pm 0.282$ & $2.65 \pm 0.034$ & $3.73 \pm 0.049$ & $5.48 \pm 0.076$ \\
\hline & Improvement rate $(\%)$ & 8.32 & 9.71 & 5.05 & 5.02 & 7.52 & 4.92 \\
\hline
\end{tabular}

The overall performance of our proposed SSDRec and all the baselines on the three real world datasets is shown in Table 4 from which we can obtain the following observations: 1) SSDRec significantly outperforms all the baselines with at least $4.92 \%$ improvement over the second best model. 2) Static models BPR and $\mathrm{NCF}$ have much worse performance indicating the interests of developers change 
frequently and it is of great importance to model the dynamics of developers' interests. 3) Models considering social influence, i.e., DGRec and SSDRec have a better performance which enhances the assumption that the social relations among developers have an important impact on the evolution of developers' interests. 4) The significant improvement of SSDRec over DGRec also indicates the important impact of dependency constraints which agrees with the practices in software development, that is, a developer usually chooses to watch new software packages based on his/her existing technical stacks. 5) SSDRec is best suited to the PHP dataset. The reason may be that PHP is a dedicated programming language for backend web development while Ruby and JavaScript have diversified application fields, and in the PHP community, developers have tight social and dependency relations and shorter session length.

\subsection{Ablation Studies (RQ2)}

As SSDRec is composed of several components, we conduct the ablation studies by comparing the performance of the variants of SSDRec to demonstrate the effectiveness of different components.

\subsubsection{Effect of social network and dependency network (RQ2(a))}

SSDRec utilizes two graph attention networks to capture the social influence among developers and dependency constraints among software packages. To illustrate their impact on the recommendation performance, we compare the performance of the two variants of SSDRec, i.e., SSDRec-social and SSDRec-dependency with SSDRec. SSDRec-social and SSDRec-dependency exclude the graph attention network for dependency network and social network, respectively. Actually, SSDRec-social only considers the social influence and is identical to DGRec. The detailed modifications of SSDRec variants are shown in Table 5 . 
Table 5: Detailed modifications of SSDRec variants. $W_{T}$ is the transformation matrix to transform the dimension of developer to $E$.

\begin{tabular}{lr}
\hline Variant & \multicolumn{1}{c}{ Modification } \\
\hline SSDRec-social & Eq. $40 \rightarrow \mathbf{e}_{i}=\mathbf{e}_{i}^{(0)}$ \\
SSDRec-dependency & Eq. $11 \rightarrow \mathbf{s}_{u, T}=W_{T} \cdot \mathbf{s}_{u}^{(0)}$ \\
\hline
\end{tabular}

Table 6: Performance of SSDRec and its variants concerning about social influence or dependency constraints.

\begin{tabular}{|c|c|c|c|c|c|c|c|}
\hline \multirow{2}{*}{ Dataset } & \multirow{2}{*}{ Model } & \multicolumn{3}{|c|}{$H R @ K(\%)$} & \multicolumn{3}{|c|}{$N D C G @ \mathrm{~K}(\%)$} \\
\hline & & 10 & 20 & 50 & 10 & 20 & 50 \\
\hline \multirow{3}{*}{ PHP } & SSDRec-social & $11.07 \pm 0.040$ & $17.29 \pm 0.020$ & $29.64 \pm 0.069$ & $5.37 \pm 0.024$ & $7.04 \pm 0.025$ & $9.55 \pm 0.015$ \\
\hline & SSDRec-dependency & $11.07 \pm 0.061$ & $17.70 \pm 0.043$ & $29.89 \pm 0.080$ & $5.30 \pm 0.036$ & $7.05 \pm 0.042$ & $9.53 \pm 0.034$ \\
\hline & SSDRec & $11.95 \pm 0.081$ & $18.93 \pm 0.135$ & $31.13 \pm 0.077$ & $5.88 \pm 0.042$ & $7.74 \pm 0.020$ & $10.22 \pm 0.005$ \\
\hline \multirow{3}{*}{ Ruby } & SSDRec-social & $7.00 \pm 0.027$ & $10.86 \pm 0.027$ & $20.73 \pm 0.058$ & $3.68 \pm 0.006$ & $4.70 \pm 0.001$ & $6.68 \pm 0.017$ \\
\hline & SSDRec-dependency & $7.25 \pm 0.111$ & $10.69 \pm 0.099$ & $19.76 \pm 0.473$ & $3.74 \pm 0.024$ & $4.65 \pm 0.025$ & $6.44 \pm 0.109$ \\
\hline & SSDRec & $7.63 \pm 0.169$ & $12.05 \pm 0.072$ & $21.98 \pm 0.115$ & $4.01 \pm 0.047$ & $5.19 \pm 0.039$ & $7.17 \pm 0.045$ \\
\hline \multirow{3}{*}{ JavaScript } & SSDRec-social & $5.26 \pm 0.046$ & $8.87 \pm 0.061$ & $17.58 \pm 0.065$ & $2.53 \pm 0.005$ & $3.47 \pm 0.024$ & $5.22 \pm 0.031$ \\
\hline & SSDRec-dependency & $4.68 \pm 0.072$ & $8.04 \pm 0.037$ & $15.73 \pm 0.071$ & $2.28 \pm 0.064$ & $3.14 \pm 0.046$ & $4.69 \pm 0.058$ \\
\hline & SSDRec & $5.70 \pm 0.085$ & $9.73 \pm 0.149$ & $18.47 \pm 0.282$ & $2.65 \pm 0.034$ & $3.73 \pm 0.049$ & $5.48 \pm 0.076$ \\
\hline
\end{tabular}

The results are shown in Table 6. SSDRec has better performance than both of its variants, which validates the effectiveness of the composition of both components in our model. Moreover, it can also be found that social influence and dependency constraints have different impacts in different development communities. In PHP community, SSDRec-dependency usually outperforms SSDRec-social while in Ruby and JavaScript communities it is just the opposite.

\subsubsection{Effect of dynamic interests and static interests (RQ2(b))}

In the software development community, a developer is generally specialized in a specific technical base and gradually expand it with newer technique. For example, a frontend developer is usually specialized in JavaScript and gradually learns different frontend JavaScript frameworks as they are evolving rapidly, from jQuery to Angular to Vue.js. Thus, developers' interests can be divided into long-term static interests and short-term dynamic interests. Table 7 shows the effectiveness of capturing both dynamic and static interests for software recommendation. Especially, in PHP community and JavaScript community dynamic interests play a more important role, which is the opposite in Ruby community. This is because PHP community 
and JavaScript community evolve more rapidly and new techniques and frameworks keep emerging all the time.

Table 7: Performance of SSDRec and its variants concerning about dynamic or static interests.

\begin{tabular}{cccccccc}
\hline \multirow{2}{*}{ Dataset } & Model & \multicolumn{3}{c}{ HR@K(\%) } & \multicolumn{3}{c}{ NDCG@K(\%) } \\
& & 10 & 20 & 50 & 10 & 20 \\
\hline \multirow{2}{*}{ PHP } & SSDRec-dynamic & $11.37 \pm 0.011$ & $18.04 \pm 0.098$ & $30.83 \pm 0.202$ & $5.68 \pm 0.014$ & $7.46 \pm 0.047$ & $10.05 \pm 0.056$ \\
& SSDRec-static & $11.03 \pm 0.036$ & $17.62 \pm 0.109$ & $30.00 \pm 0.055$ & $5.44 \pm 0.028$ & $7.23 \pm 0.031$ & $9.73 \pm 0.013$ \\
& SSDRec & $\mathbf{1 1 . 9 5} \pm \mathbf{0 . 0 8 1}$ & $\mathbf{1 8 . 9 3} \pm \mathbf{0 . 1 3 5}$ & $\mathbf{3 1 . 1 3} \pm \mathbf{0 . 0 7 7}$ & $\mathbf{5 . 8 8} \pm \mathbf{0 . 0 4 2}$ & $\mathbf{7 . 7 4} \pm \mathbf{0 . 0 2 0}$ & $\mathbf{1 0 . 2 2} \pm \mathbf{0 . 0 0 5}$ \\
\hline \multirow{2}{*}{ Ruby } & SSDRec-dynamic & $7.30 \pm 0.527$ & $11.34 \pm 0.565$ & $21.29 \pm 0.416$ & $3.77 \pm 0.265$ & $4.81 \pm 0.270$ & $6.82 \pm 0.227$ \\
& SSDRec-static & $7.39 \pm 0.296$ & $11.36 \pm 0.085$ & $21.01 \pm 0.099$ & $3.84 \pm 0.067$ & $4.88 \pm 0.315$ & $6.81 \pm 0.086$ \\
& SSDRec & $\mathbf{7 . 6 3} \pm \mathbf{0 . 1 6 9}$ & $\mathbf{1 2 . 0 5} \pm \mathbf{0 . 0 7 2}$ & $\mathbf{2 1 . 9 8} \pm \mathbf{0 . 1 1 5}$ & $\mathbf{4 . 0 1} \pm \mathbf{0 . 0 4 7}$ & $\mathbf{5 . 1 9} \pm \mathbf{0 . 0 3 9}$ & $\mathbf{7 . 1 7} \pm \mathbf{0 . 0 4 5}$ \\
\hline \multirow{2}{*}{ JavaScript } & SSDRec-dynamic & $5.54 \pm 0.169$ & $9.50 \pm 0.038$ & $18.23 \pm 0.068$ & $2.58 \pm 0.005$ & $3.62 \pm 0.032$ & $5.37 \pm 0.005$ \\
& SSDRec-static & $5.10 \pm 0.042$ & $8.67 \pm 0.031$ & $16.76 \pm 0.195$ & $2.36 \pm 0.003$ & $3.30 \pm 0.038$ & $4.92 \pm 0.034$ \\
& SSDRec & $\mathbf{5 . 7 0} \pm \mathbf{0 . 0 8 5}$ & $\mathbf{9 . 7 3} \pm \mathbf{0 . 1 4 9}$ & $\mathbf{1 8 . 4 7} \pm \mathbf{0 . 2 8 2}$ & $\mathbf{2 . 6 5} \pm \mathbf{0 . 0 3 4}$ & $\mathbf{3 . 7 3} \pm \mathbf{0 . 0 4 9}$ & $\mathbf{5 . 4 8} \pm \mathbf{0 . 0 7 6}$ \\
\hline
\end{tabular}

\subsection{Hyperparameter Analysis (RQ3)}

To ensure the flexibility of the proposed SSDRec, it employs several hyperparameters. In this section, we conduct experiments to show how the hyperparameters affect the performance of our model.

\subsubsection{Neighborhood sample size}

Due to the heterogeneity of both social and dependency networks, we utilize the sampling technique proposed in [39] to ensure the training efficiency of the two graph attention networks. $\left|N_{S}\right|\left(\left|N_{D}\right|\right)$ neighbors are sampled for the first layer of the graph attention network for social network (dependency network) and the neighborhood sample size of each layer is half of the previous layer. In the analysis, we measure the recommendation performance using $H R @ 10$ and $N D C G @ 10$ under different neighborhood sample sizes as shown in Figure 6. 


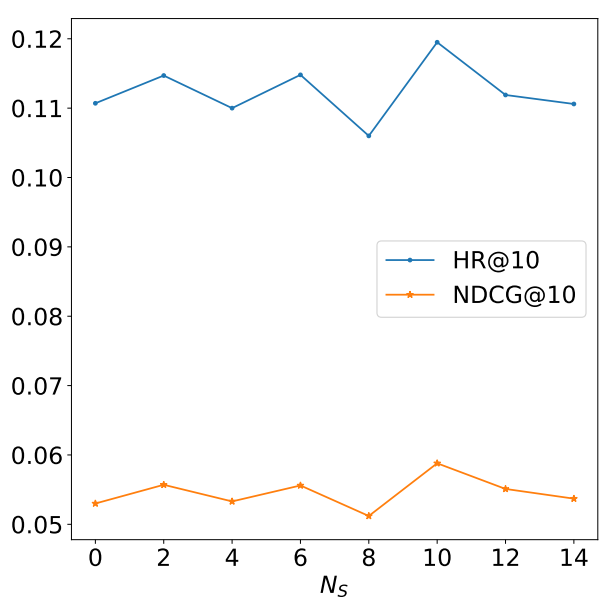

(a) PHP-social

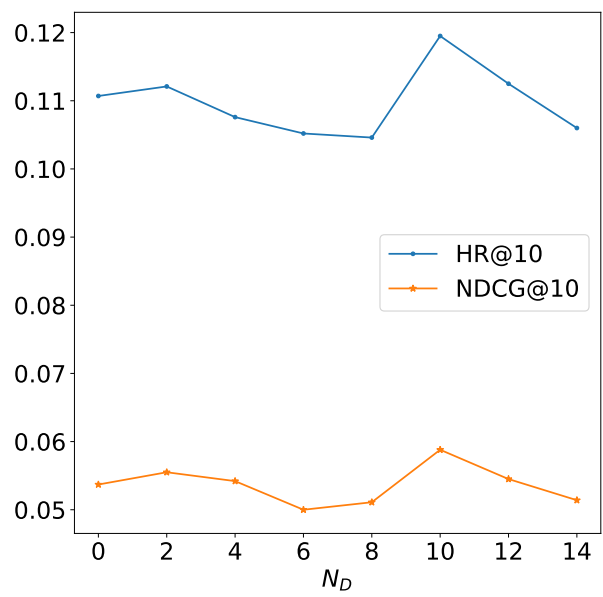

(c) PHP-dependency

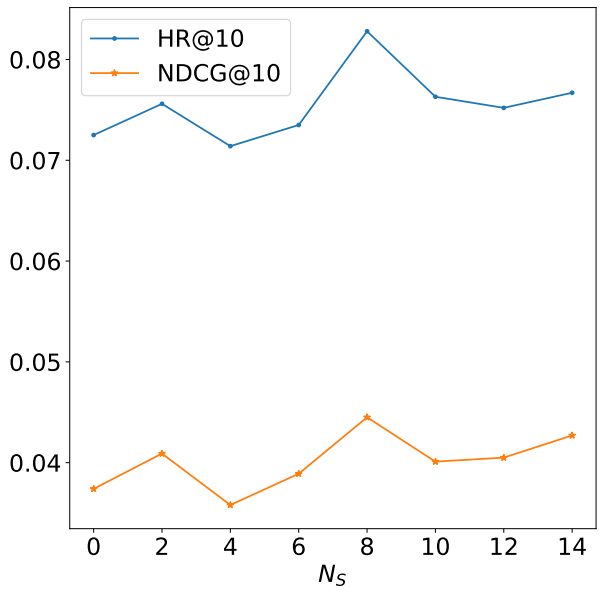

(b) Ruby-social

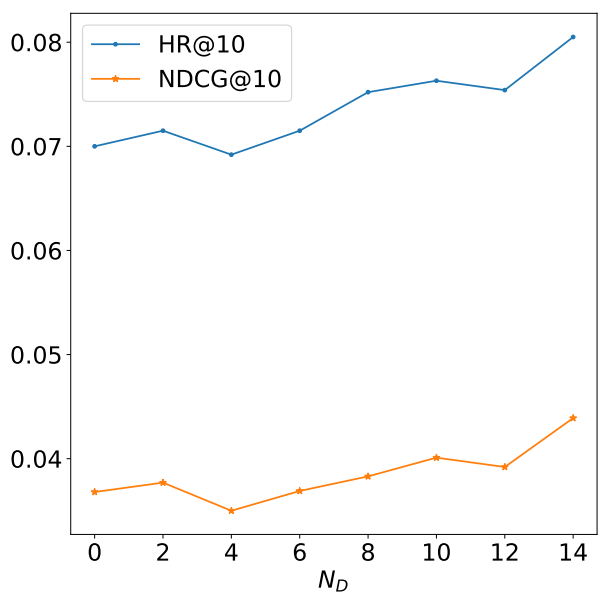

(d) Ruby-dependency

Figure 6: Impact of neighborhood sample size.

From Figure 6, it can be found that neighborhood sample size for both graph attention networks for social and dependency networks has an impact on the recommendation performance, and too large or too small neighborhood sample size will both decrease the performance. The optimal neighbor sample sizes $N_{S}$ and $N_{D}$ for PHP are both 10, while they are 8 and 14 for Ruby, respectively.

\subsubsection{Session length}

Developers usually focus on a specific technical field during a certain length of time period, which is modeled as a session. Session length determines the granularity of dynamic interest modeling. In this analysis, we demonstrate the impact of session 
length by comparing the performance of SSDRec with different session lengths. From the result shown in Figure 7, it can be observed that the performance decreases with the increase of session length and finally lies in a steady state. The reason is that longer session length means more coarse-grained granularity of dynamic interest. In an extreme setting where the whole interactions of a developer are segmented into one session, the RNN component of SSDRec captures the long-term static interest of the developer instead of short-term dynamic interest.

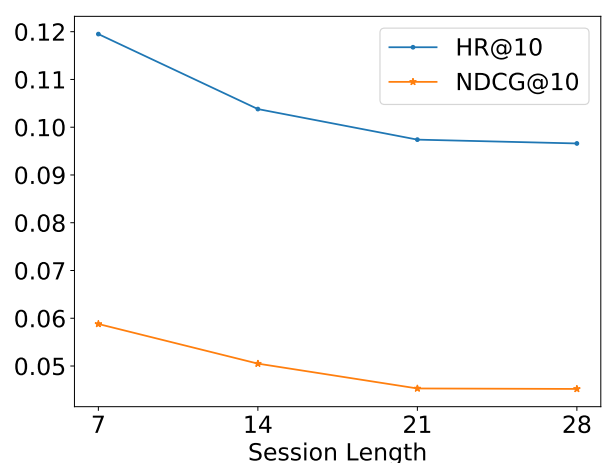

(a) PHP

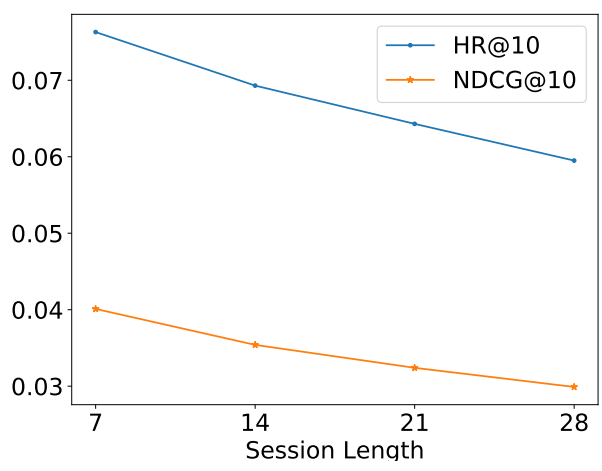

(b) Ruby

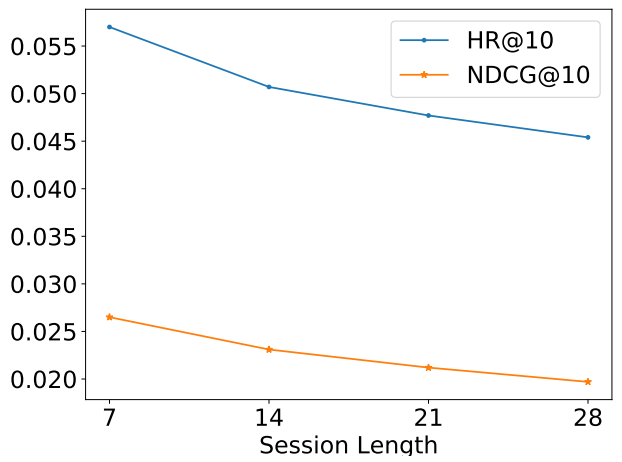

(c) JavaScript

Figure 7: Impact of session length.

\subsection{Attention Visualization}

Section 5.4 .2 has demonstrated session can capture fine-grained interests. In this section, we further verify the hypothesis that developers' interests are relatively stable within a session but evolve across sessions by visualizing the attention weights of our model from the perspectives of both the overall development community and individual developer. 


\subsubsection{The overall development community}

The attention weights between each pair of nodes of the social network across all sessions are grouped and the variance is then calculated as the inter-session attention variance. Similarly, the intra-session attention variance is calculated within each session. The distributions of both intra- and inter-session attention variance for PHP, Ruby and JavaScript are shown in Figure 8 from which we can observe that the attention weights across sessions vary largely than that within sessions. This confirms that developers' interests are relatively stable within a session but evolve across sessions.

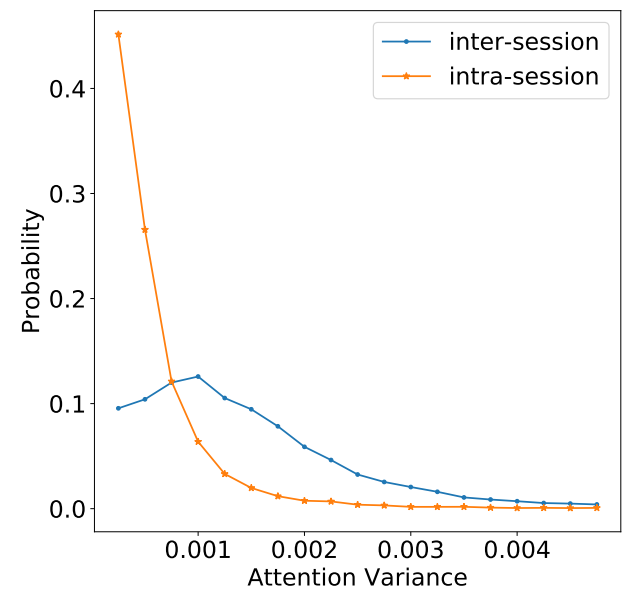

(a) PHP

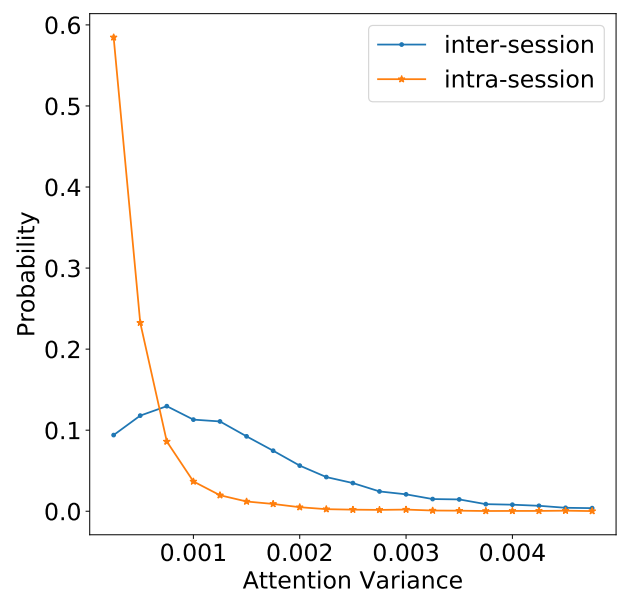

(b) Ruby

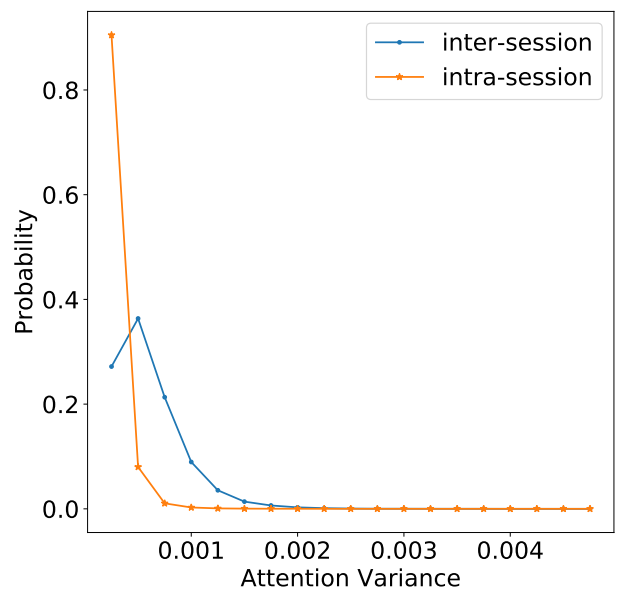

(c) JavaScript

Figure 8: Attention variance distributions of inter-session and intra-session 


\subsubsection{Individual developer}

In this section, we conduct a case study of an individual developer $u 1039$ to show his/her behaviors within and across sessions. This developer has 8 test sessions and at least 5 friends in PHP community and the visualization results are shown in Figure 9. From the results, the following observations can be found:

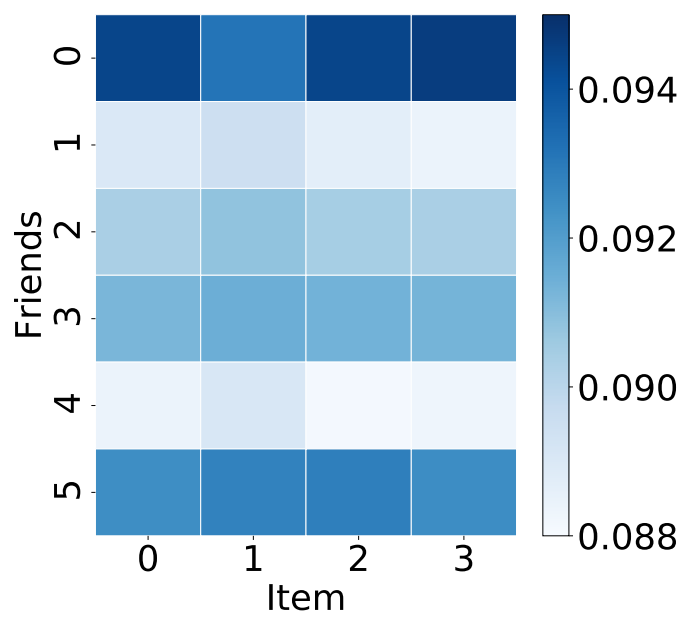

(a) intra-session attention

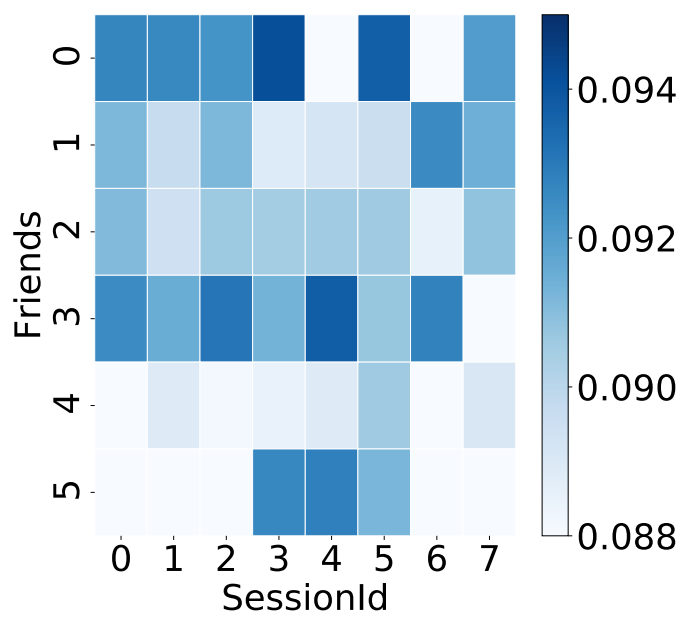

(b) inter-session attention

Figure 9: Intra- and inter-session attention visualization for developer $u 1039$.

(1) The developer's interest is generally stable within the session and his/her technical choices are mainly influenced by friends 0 and 5 .

(2) The developer's interest evolves across sessions. During the first three sessions, i.e., session 0, 1 and 2, the developer's technical choices are mainly influenced by friends 0 and 3 while then in the three sessions that follow friend 5 begins to influence him/her. Maybe the developer begins to pay attention to a new technical field in session 3. Finally, the influence of friend 5 disappears in the last two sessions. The developer may leave this technical field after some investigation.

\section{Conclusions}

In this article, we study the software recommendation problem and propose the Session-based Social and Dependency-aware software Recommendation model SSDRec to model the dynamic interests of developers with both social influence and dependency constraints in a unified framework. Especially, an RNN is employed to 
model the short-term dynamic interests and two GATs are utilized to capture social influence from friends and dependency constraints from dependent software packages, respectively. The experiments on real world datasets verify the effectiveness of all three components of our model. In the future, we will consider higher order relations in both social and dependency networks.

\section{CRediT authorship contribution statement}

Dengcheng Yan: Conceptualization, Methodology, Writing - Original Draft. Tianyi Tang: Software, Validation, Data Curation, Writing - Original Draft. Wenxin Xie: Software, Validation. Yiwen Zhang: Supervision, Writing - review \& editing. Qiang He: Writing - review \& editing.

\section{Declaration of Competing Interest}

The authors declare that they have no known competing financial interests or personal relationships that could have appeared to influence the work reported in this paper.

\section{Acknowledgement}

This work is supported by the National Key Research and Development Program of China (No. 2019YFB1704101), the National Natural Science Foundation of China (Grant No. 61872002, U1936220) and the University Natural Science Research Project of Anhui Province (Grant No. KJ2019A0037).

\section{References}

[1] Steffen Rendle, Christoph Freudenthaler, Zeno Gantner, and Lars SchmidtThieme. Bpr: Bayesian personalized ranking from implicit feedback. In Proceedings of the Twenty-Fifth Conference on Uncertainty in Artificial Intelligence, UAI '09, page 452-461. AUAI Press, 2009.

[2] Xiangnan He, Lizi Liao, Hanwang Zhang, Liqiang Nie, Xia Hu, and Tat-Seng Chua. Neural collaborative filtering. In Proceedings of the 26th International 
Conference on World Wide Web, WWW'17, page 173-182. International World Wide Web Conferences Steering Committee, 2017.

[3] Hanhua Chen, Hai Jin, and Shaoliang Wu. Minimizing inter-server communications by exploiting self-similarity in online social networks. IEEE Transactions on Parallel and Distributed Systems, 27(4):1116-1130, 2016.

[4] Yiwe Zhang, Jie Pan, Lianyong Qi, and Qiang He. Privacy-preserving quality prediction for edge-based iot services. Future Generation Computer Systems, 114:336-348, 2021.

[5] Yiwen Zhang, Kaibin Wang, Qiang He, Feifei Chen, Shuiguang Deng, Zibin Zheng, and Yun Yang. Covering-based web service quality prediction via neighborhood-aware matrix factorization. IEEE Transactions on Services Computing, pages 1-1, 2019.

[6] Makoto Ichii, Yasuhiro Hayase, Reishi Yokomori, Tetsuo Yamamoto, and Katsuro Inoue. Software component recommendation using collaborative filtering. In Proceedings of the 2009 ICSE Workshop on Search-Driven DevelopmentUsers, Infrastructure, Tools and Evaluation, SUITE '09, page 17-20. IEEE Computer Society, 2009.

[7] Ferdian Thung, David Lo, and Julia Lawall. Automated library recommendation. In 2013 20th Working Conference on Reverse Engineering (WCRE), pages 182-191, 2013.

[8] Qiang He, Bo Li, Feifei Chen, John Grundy, Xin Xia, and Yun Yang. Diversified third-party library prediction for mobile app development. IEEE Transactions on Software Engineering, 2020.

[9] Youhua Jiang, Siyi Yan, Peng Qi, and Yan Sun. Adapting to user interest drifts for recommendations in scratch. In 2020 International Wireless Communications and Mobile Computing (IWCMC), pages 1528-1534, 2020.

[10] Chen Cheng, Haiqin Yang, Michael R. Lyu, and Irwin King. Where you like to go next: Successive point-of-interest recommendation. In Proceedings of the 
Twenty-Third International Joint Conference on Artificial Intelligence, IJCAI '13, page 2605-2611. AAAI Press, 2013.

[11] Chang Li, Dongjin Song, and Dacheng Tao. Multi-task recurrent neural networks and higher-order markov random fields for stock price movement prediction: Multi-task rnn and higer-order mrfs for stock price classification. In Proceedings of the 25th ACM SIGKDD International Conference on Knowledge Discovery $\&$ Data Mining, KDD '19, page 1141-1151. Association for Computing Machinery, 2019.

[12] Yequan Wang, Aixin Sun, Jialong Han, Ying Liu, and Xiaoyan Zhu. Sentiment analysis by capsules. In Proceedings of the 2018 World Wide Web Conference, WWW'18, page 1165-1174. International World Wide Web Conferences Steering Committee, 2018.

[13] Jarana Manotumruksa, Craig Macdonald, and Iadh Ounis. A deep recurrent collaborative filtering framework for venue recommendation. In Proceedings of the 2017 ACM on Conference on Information and Knowledge Management, CIKM '17, page 1429-1438. Association for Computing Machinery, 2017.

[14] Disheng Dong, Xiaolin Zheng, Ruixun Zhang, and Yan Wang. Recurrent collaborative filtering for unifying general and sequential recommender. In Proceedings of the Twenty-Seventh International Joint Conference on Artificial Intelligence, IJCAI-18, pages 3350-3356. International Joint Conferences on Artificial Intelligence Organization, 2018.

[15] Balázs Hidasi, Alexandros Karatzoglou, Linas Baltrunas, and Domonkos Tikk. Session-based recommendations with recurrent neural networks. In International Conference on Learning Representations., 2016.

[16] Shoujin Wang, Liang Hu, Yan Wang, Quan Z. Sheng, Mehmet Orgun, and Longbing Cao. Modeling multi-purpose sessions for next-item recommendations via mixture-channel purpose routing networks. In Proceedings of the TwentyEighth International Joint Conference on Artificial Intelligence, pages 3771- 
3777. International Joint Conferences on Artificial Intelligence Organization, 2019.

[17] Massimiliano Ruocco, Ole Steinar Lillestøl Skrede, and Helge Langseth. Intersession modeling for session-based recommendation. In Proceedings of the 2nd Workshop on Deep Learning for Recommender Systems, DLRS 2017, page 24-31. Association for Computing Machinery, 2017.

[18] Hao Ma, Dengyong Zhou, Chao Liu, Michael R. Lyu, and Irwin King. Recommender systems with social regularization. In Proceedings of the Fourth ACM International Conference on Web Search and Data Mining, WSDM '11, page 287-296. Association for Computing Machinery, 2011.

[19] Tong Zhao, Julian McAuley, and Irwin King. Leveraging social connections to improve personalized ranking for collaborative filtering. In Proceedings of the 23rd ACM International Conference on Conference on Information and Knowledge Management, CIKM '14, page 261-270. Association for Computing Machinery, 2014.

[20] Yue Ding, Dong Wang, Xin Xin, Guoqiang Li, Daniel Sun, Xuezhi Zeng, and Rajiv Ranjan. Scfm: Social and crowdsourcing factorization machines for recommendation. Applied Soft Computing, 66:548-556, 2018.

[21] Shuiguang Deng, Longtao Huang, Guandong Xu, Xindong Wu, and Zhaohui Wu. On deep learning for trust-aware recommendations in social networks. IEEE Transactions on Neural Networks and Learning Systems, 28(5):1164$1177,2017$.

[22] Xiang Wang, Xiangnan He, Meng Wang, Fuli Feng, and Tat-Seng Chua. Neural graph collaborative filtering. In Proceedings of the 42nd International ACM SIGIR Conference on Research and Development in Information Retrieval, SIGIR'19, page 165-174. Association for Computing Machinery, 2019.

[23] Petar Veličković, Guillem Cucurull, Arantxa Casanova, Adriana Romero, Pietro 
Liò, and Yoshua Bengio. Graph attention networks. In International Conference on Learning Representations, 2018.

[24] Tian Bian, Xi Xiao, Tingyang Xu, Peilin Zhao, Wenbing Huang, Yu Rong, and Junzhou Huang. Rumor detection on social media with bi-directional graph convolutional networks. In Proceedings of the AAAI Conference on Artificial Intelligence, volume 34, pages 549-556, 2020.

[25] Wenqi Fan, Yao Ma, Qing Li, Yuan He, Eric Zhao, Jiliang Tang, and Dawei Yin. Graph neural networks for social recommendation. In The World Wide Web Conference, WWW'19, page 417-426. Association for Computing Machinery, 2019.

[26] Weiping Song, Zhiping Xiao, Yifan Wang, Laurent Charlin, Ming Zhang, and Jian Tang. Session-based social recommendation via dynamic graph attention networks. In Proceedings of the Twelfth ACM International Conference on Web Search and Data Mining, WSDM '19, page 555-563. Association for Computing Machinery, 2019.

[27] Nuri Almarimi, Ali Ouni, Salah Bouktif, Mohamed Wiem Mkaouer, Raula Gaikovina Kula, and Mohamed Aymen Saied. Web service api recommendation for automated mashup creation using multi-objective evolutionary search. Applied Soft Computing, 85:105830, 2019.

[28] Yiwen Zhang, Yin Chunhui, Wu Qilin, He Qiang, Zhu, and Haibin. Locationaware deep collaborative filtering for service recommendation. IEEE Transactions on Systems, Man, and Cybernetics: Systems, pages 1-12, 2019.

[29] Ido Guy, Naama Zwerdling, David Carmel, Inbal Ronen, Erel Uziel, Sivan Yogev, and Shila Ofek-Koifman. Personalized recommendation of social software items based on social relations. In Proceedings of the Third ACM Conference on Recommender Systems, RecSys '09, page 53-60. Association for Computing Machinery, 2009.

[30] Georgios Gousios. The ghtorent dataset and tool suite. In Proceedings of the 
10th Working Conference on Mining Software Repositories, MSR '13, pages 233-236. IEEE Press, 2013.

[31] Chunyang Chen and Zhenchang Xing. SimilarTech: Automatically recommend analogical libraries across different programming languages. In 2016 31st IEEE/ACM International Conference on Automated Software Engineering (ASE), pages 834-839, 2016.

[32] Jyun-Yu Jiang, Pu-Jen Cheng, and Wei Wang. Open source repository recommendation in social coding. In Proceedings of the 40th International ACM SIGIR Conference on Research and Development in Information Retrieval, pages 1173-1176, 2017.

[33] Xiaobing Sun, Wenyuan Xu, Xin Xia, Xiang Chen, and Bin Li. Personalized project recommendation on github. Science China Information Sciences, 61(5):1-14, 2018.

[34] Zhensu Sun, Yan Liu, Ziming Cheng, Chen Yang, and Pengyu Che. Req2Lib: A semantic neural model for software library recommendation. In 2020 IEEE 27 th International Conference on Software Analysis, Evolution and Reengineering (SANER), pages 542-546, 2020.

[35] Pengcheng Zhang, Fang Xiong, Hareton K.N. Leung, and Wei Song. FunkRpDAE: Personalized project recommendation using deep learning. IEEE Transactions on Emerging Topics in Computing, 9(2):886-900, 2021.

[36] Cheng Yang, Qiang Fan, Tao Wang, Gang Yin, Xun-hui Zhang, Yue Yu, and Hua-min Wang. RepoLike: amulti-feature-based personalized recommendation approach for open-source repositories. Frontiers of Information Technology 8 Electronic Engineering, 20(2):222-237, 2019.

[37] Martín Abadi, Paul Barham, Jianmin Chen, Zhifeng Chen, Andy Davis, Jeffrey Dean, Matthieu Devin, Sanjay Ghemawat, Geoffrey Irving, Michael Isard, Manjunath Kudlur, Josh Levenberg, Rajat Monga, Sherry Moore, Derek G. Murray, Benoit Steiner, Paul Tucker, Vijay Vasudevan, Pete Warden, Martin 
Wicke, Yuan Yu, and Xiaoqiang Zheng. Tensorflow: A system for large-scale machine learning. In Proceedings of the 12th USENIX Conference on Operating Systems Design and Implementation, OSDI'16, page 265-283. USENIX Association, 2016.

[38] Diederik P Kingma and Jimmy Ba. Adam: A method for stochastic optimization. In International Conference on Learning Representations, 2014.

[39] William L. Hamilton, Rex Ying, and Jure Leskovec. Inductive representation learning on large graphs. In Proceedings of the 31st International Conference on Neural Information Processing Systems, NIPS'17, page 1025-1035. Curran Associates Inc., 2017. 